\title{
Phase transitions of perfluorocarbon nanoemulsion induced with ultrasound: A mathematical model
}

\author{
William G. Pitt ${ }^{\mathrm{a}}$, , Ram N. Singh ${ }^{\text {a }}$, Krystian X. Perez ${ }^{\mathrm{a}}$, Ghaleb A. Husseini ${ }^{\mathrm{b}}$, Daniel R. Jack ${ }^{\mathrm{a}}$ \\ ${ }^{a}$ Chemical Engineering Department, Brigham Young University, Provo, UT, USA \\ ${ }^{\mathrm{b}}$ Chemical Engineering Department, American University of Sharjah, Sharjah, United Arab Emirates \\ https://doi.org/10.1016/j.ultsonch.2013.08.005
}

While ultrasound has been used in many medical and industrial applications, only recently has research been one on phase transformations induced by ultrasound. This paper presents a numerical model and the predicted results of the phase transformation of a spherical nanosized droplet of perfluorocarbon in water. Such a model has applications in acoustic droplet vaporization, the generation of gas bubbles for medical imaging, therapeutic delivery and other biomedical applications.

The formation of a gas phase and the subsequent bubble dynamics were studied as a function of acoustic parameters, such as frequency and amplitude, and of the physical aspects of the perfluorocarbon nanodroplets, such as chemical species, temperature, droplet size and interfacial energy. The model involves simultaneous applications of mass, energy and momentum balances to describe bubble formation and collapse, and was developed and solved numerically. It was found that, all other parameters being constant, the maximum bubble size and collapse velocity increases with increasing ultrasound amplitude, droplet size, vapor pressure and temperature. The bubble size and collapse velocity decreased with increasing surface tension and frequency. These results correlate with experimental observations of acoustic droplet vaporization.

\section{Introduction}

Ultrasound (US) is a very effective modality for biomedical imaging and therapeutic delivery because it can be non-invasively focused to interrogate a region or to deliver energy to a desired tissue volume. In drug delivery, the unique advantages of ultrasound make it convenient for localized delivery to tumors and for treating specialized organs such as the heart, eyes, and brain [1-10]. Moreover, ultrasound exposure has been shown to induce gaps between endothelial cells [11,12] and widen epithelial intercellular spaces, which leads to extravasation of drug carriers to targeted tissues [13-17].

The underlying mechanism behind the physiological consequences of US is acoustic cavitation - the formation and subsequent oscillation of bubbles in an ultrasonic field. Ultrasonic acoustic cavitation of bubbles perturbs the cell integrity structure by several mechanisms, and thus increases its permeability to drugs and other solutes. For example, cavitation can transiently disrupt the cell membrane and forms pores therein [18-22].

Unfortunately gas bubbles (about $1-10 \mathrm{~lm}$ in diameter) are too large to penetrate through the walls of capillaries in tumors to the tissue beyond, and thus this type of ultrasonic-induced therapy is limited to the surface and internal volume of the circulatory 
system. However, it is desired to extend imaging and therapeutic delivery to regions beyond the arteries, veins, and capillaries. The former two vessels are impermeable to contrast agents because of the thick layers of tightly packed cells and connective tissues. However, capillaries consist of only a single layer of endothelial cells. Yet in most tissues these cells are tightly connected to each other, so gas bubbles are not able to penetrate the tissue. However, many types of tumors display disruption of the normal endothelial covering of the capillary wall, and thus vesicles of less than $300 \mathrm{~nm}$ can penetrate beyond the capillaries [24-26].

Stabilized liquid perfluorocarbon (PFC) droplets have been formulated and evaluated as contrast agents for in vivo diagnostic and therapeutic purposes [27-34]. Many can be produced with diameters of less than $600 \mathrm{~nm}$. One goal of our research is to exploit physics and chemistry principles in a novel method whereby ultrasound is used to produce cavitation bubbles beyond the capillary wall from emulsion droplets of low-boiling-point PFCs having a size that can penetrate through the leaky capillaries of many tumors. More specifically the purpose of the research reported herein is to understand the physics and chemistry of phase transition of perfluoropentane $\left(\mathrm{PFC}_{5}\right)$ and perfluorohexane $\left(\mathrm{PFC}_{6}\right)$ nanoemulsions subjected to $20-\mathrm{kHz}$ and $500-\mathrm{kHz}$ ultrasound frequencies. This phase-transition phenomenon has recently been labeled acoustic droplet vaporization (ADV) $[10,28,35-37]$.

A liquid at constant temperature may be subject to a decreasing pressure, $\mathrm{P}_{1}$, which eventually falls below its vapor pressure $\left(\mathrm{P}_{\text {vap }}\right)$. The process of rupturing a liquid by decreasing the pressure at constant liquid temperature is also called cavitation and is a common topic of hydraulics. Fig. 1 illustrates the local pressure variation as ultrasound propagates in a liquid medium. There are positive and negative pressure fluctuations (relative to the local hydrostatic pressure) that oscillate around atmospheric pressure. When the rarefaction cycle of the acoustic wave decreases the local pressure to values below the vapor pressure of the PFC liquid for sufficient time, it boils (evaporates) and forms gas bubbles. Sufficient time means that the local pressure is lower than the vapor pressure for the time needed to produce heterogeneous or homogeneous nucleation of the gas phase. Water vapor pressure is $3170 \mathrm{~Pa}$ at $25 \mathrm{C}$, an order of magnitude lower than the vapor pressure of a small PCF such as perfluoropentane, and thus the $\mathrm{PFC}_{5}$ bubbles will form before homogeneous nucleation of water vapor bubbles. However, that bubble will contain both PFC vapor and water vapor, since some water will evaporate into it. A bubble point calculation reveals that as the local pressure drops, the first perfluoropentane bubble to form contains only $3.5 \%$ and $4.4 \%$ water vapor at equilibrium at $25 \mathrm{C}$ and $37 \mathrm{C}$, respectively. Perfluorohexane has a lower vapor pressure, so there is slightly more water in the first bubble formed: $9.8 \%$ and $11.6 \%$ water vapor at $25 \mathrm{C}$ and $37 \mathrm{C}$, respectively.

The pressure inside a nanoemulsion droplet is greater than the surrounding liquid pressure because of the additional contribution from the Laplace pressure: $\Delta P_{L P}=2 \gamma / r$, where $\gamma$ is interfacial energy, and $\mathrm{r}$ is the radius of the emulsion droplet. As the sum of the local pressure and the Laplace pressure drops below the vapor pressure of the liquid PFC, there is a subpressurization driving potential to nucleate the gas phase. Analogous to subcooling of liquids (local temperature is below the freezing point without solid formation), we define subpressurization as the difference between the vapor pressure and the local pressure. However, if the acoustic cycle is too short or the subpressurization is inadequate, no gas phase will form. While there are reports of rapid gas phase formation at very large values of subpressurization [38-40], experimental observation of the kinetics of gas nucleation from PFC droplets at small subpressurizations are not yet found in literature, so one can only speculate regarding the length of time or the subpressurization required to nucleate the gas phase [41]. To our knowledge no research has been published regarding the details of the physics of the dynamic phase transformation of perfluorocarbons (PFC) emulsion droplets by application of ultrasound. Fluorocarbons are ideal compounds for use in medical ultrasound because they have low solubility in water and are non-toxic. The PFCs that were investigated in this study are $\mathrm{PFC}_{5}$ and $\mathrm{PFC}_{6}$. At sea level $\mathrm{PFC}_{5}$ boils at $29 \mathrm{C}$ [42], and $\mathrm{PFC}_{6}$ boils at $56 \mathrm{C}[42,43]$. The former has a boiling point below body temperature 
(37 C) and the latter above body temperature. In the presence of a stabilizing surfactant, nanoemulsions are formed that are sufficiently small that the contribution of the Laplace pressure prevents the droplets from vaporizing even if they are at a temperature slightly above the boiling point [31,44-46]. However, a superheated droplet can be vaporized by ultrasound into a larger volume bubble that is stable and will not return to the liquid emulsion state because its larger diameter reduces the contribution of the Laplace pressure [10,35,38,44,47].

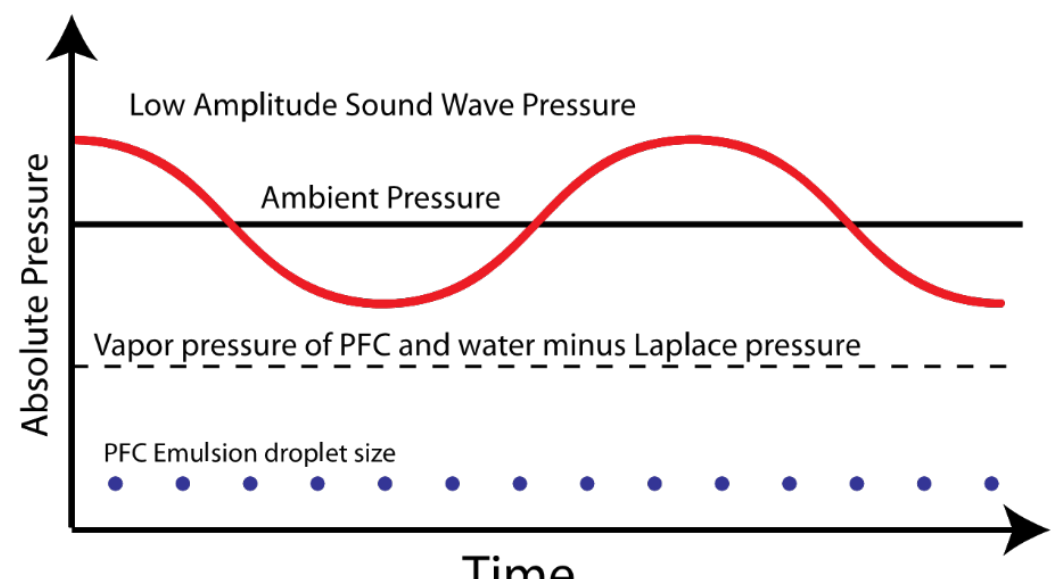

Time

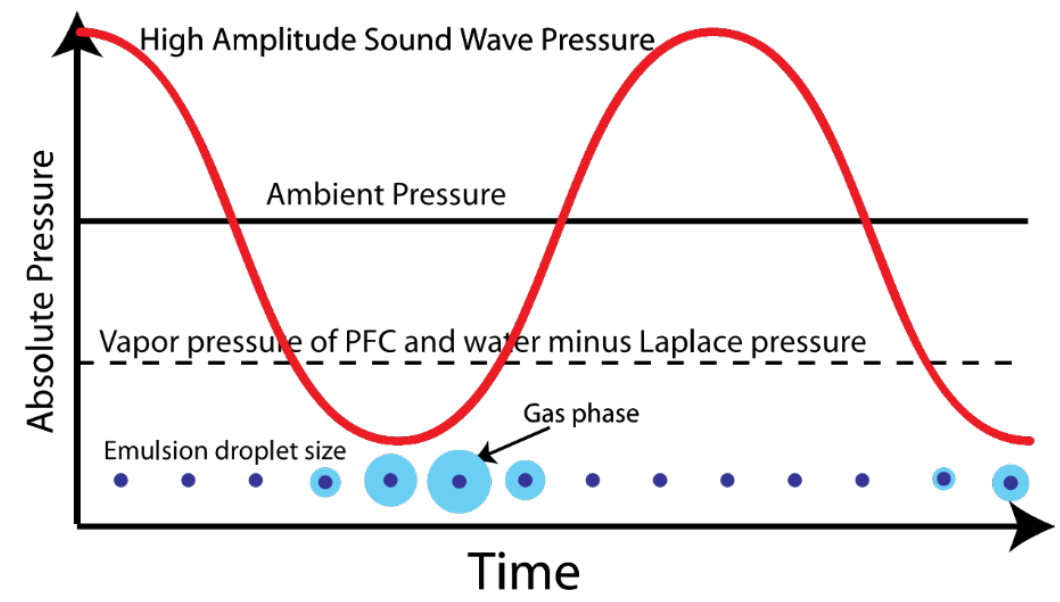

Fig. 1. Schematic (not to scale) of an ultrasonic acoustic wave in a perfluorocarbon emulsion. The upper figure illustrates an acoustic wave whose lowest pressure remains above the vapor pressure of the PFC minus the Laplace pressure. The lower figure illustrates how a gas phase forms around the emulsion droplet when the local wave pressure drops below the vapor pressure of the PFC minus the Laplace pressure. The maximum expansion of the gas is not in phase with the minimum acoustic pressure because of the momentum of the water surrounding the bubble.

Once gas bubbles are formed from the liquid droplets, the gas phase will expand and contract dynamically in response to the physical forces dictated by the mass, momentum and energy balances developed in Section 2. 


\section{Mathematical model}

When an acoustic field is applied to a nanoemulsion droplet and the pressure during the cycle drops below the vapor pressure of the liquid, a gas phase can be formed. Subsequently the gas undergoes fast expansion followed eventually by a rapid collapse when the surrounding pressure begins to increase during the acoustic cycle. As the liquid evaporates to a gas, the liquid surface cools; likewise during the gas collapse, condensation of gas molecules onto the liquid surface heats the surface up again. There is also conductive heat transfer to and from the surrounding liquid in addition to this "insensible" energy transfer. The following mathematical model is developed to describe this behavior based on bubble radius, bubble wall velocity, liquid surface temperature, and vapor pressure of the PFC liquid inside the bubble at that temperature (see Fig. 2).

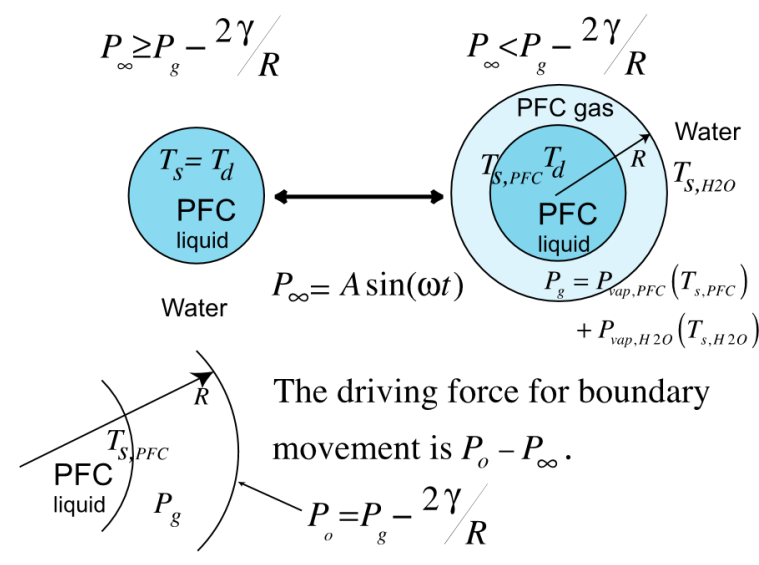

Fig. 2. Schematic diagram of the emulsion droplet (upper left) and gas phase surrounding the droplet (upper right). The gas phase is the sum of the vapor pressures $\left(\mathrm{P}_{\mathrm{vap}}\right)$ of the water and the PFC on the surface of the liquid droplet, which during expansion may be colder $\left(\mathrm{T}_{\mathrm{s}}\right)$ than the temperature at the droplet center $\left(\mathrm{T}_{\mathrm{d}}\right)$. The pressure on the water side of the gas/water interface is the vapor pressure minus the Laplace pressure, and the difference between this pressure and the acoustic driving pressure causes the gas/water interface to move.

Several assumptions are required to develop the physical model describing the dynamics of the gas bubble during ultrasonic insonation.

1. The small size of the gas phase makes pressure gradients with in the gas phase negligible. When thermal gradients exist within the gas phase, they do not create significant pressure or concentration gradients.

2. Absent other evidence, no sub-cooling is required for nucleation. Thus the bubble starts growing immediately in the first acoustic cycle as soon as the local pressure in the emulsion droplet falls below the bubble point pressure at the local temperature.

3. The gas phase exists as a spherical shell concentric with the liquid droplet between the PFC and water phases.

4. The droplet and bubble have no interaction with any other droplet or bubble; so this model pertains to a single droplet/ bubble in an infinite medium.

5. The liquids are incompressible fluids, and their kinematic viscosities are assumed to be temporally constant and spatially uniform.

6. The liquid and gas phases are Newtonian fluids, and the low pressures involved allow the gas phase to be considered ideal. The gasses form ideal mixtures with no excess volume or heat of mixing. No gasses other than water and PFC are present. 
7. $\mathrm{PFC}$ is insoluble in liquid water, and water is insoluble in liquid PFC.

The pressure acting far from the nanodroplet, $\mathrm{P}_{\infty}$, is the summation of atmospheric pressure, hydrostatic pressure, and acoustic pressure. The average atmospheric pressure $\left(\mathrm{P}_{\mathrm{atm}}\right)$ in our laboratory at Brigham Young University is about 85,783 Pa, which we used in the model to enable comparison with parallel experimental measurements [48]. The hydrostatic pressure is $\rho_{\mathrm{w}} \mathrm{gh}$, where $\mathrm{h}$ is the nanodroplet position below the water surface, $\rho_{\mathrm{w}}$ is water density, and $\mathrm{g}$ is acceleration of gravity. The current model employed a depth of $20 \mathrm{~mm}$, again to match the corresponding experimental conditions. The acoustic field causes local pressure variation described by $\mathrm{A} \sin \mathrm{xt}$, where $\mathrm{A}$ is the amplitude of the sound wave, $\mathrm{x}$ is angular velocity, and $\mathrm{t}$ is time. Before any gas phase is present, the Laplace pressure acting on the spherical nanodroplet is $2 \gamma / \mathrm{R}_{\mathrm{L}}$, where $\mathrm{c}$ is surface tension at the nanodroplet/water interface, and $\mathrm{R}_{\mathrm{L}}$ is the radius of the liquid nanodroplet, which can vary with time once a gas phase is formed. Most simulations employed a value of $3.5 \mathrm{mN} / \mathrm{m}$, the estimated energy of phosphatidylcholine-stabilized PFC in water [49]. When no gas is present, $R_{L}=R(t)$, where $R(t)$ is the distance of the water interface from the nanodroplet center. If a gas phase is present, the gas phase is in equilibrium with the liquid interfaces, so that the total pressure inside the gas phase $\left(\mathrm{P}_{\mathrm{g}}\right)$ is simply the vapor pressure of the perfluorocarbon liquid $\left(\mathrm{P}_{\text {vap, } \mathrm{PFC}}\left(\mathrm{T}_{\mathrm{s}, \mathrm{PFC}}\right)\right)$ plus the vapor pressure of the water $\left(\mathrm{Pvap}, \mathrm{H}_{2} \mathrm{O}\left(\mathrm{T}_{\mathrm{s} ;} \mathrm{H}_{2} \mathrm{O}\right)\right)$. The total pressure just outside the bubble surface $\left(\mathrm{P}_{\mathrm{o}}\right)$ is the gas-phase pressure minus the Laplace pressure, $2 \gamma / \mathrm{R}(\mathrm{t})$. The vapor pressure is only a function of the surface temperatures of the PFC liquid drop $\left(\mathrm{T}_{\mathrm{s}, \mathrm{PFC}}\right)$ and gas/water interface $\left(\mathrm{T}_{\mathrm{s}} ; \mathrm{H}_{2} \mathrm{O}\right)$. Thus when a gas phase exists, the first set of pressure equations consists of:

$$
\begin{aligned}
& \mathrm{P}_{\infty}=\mathrm{P}_{\mathrm{atm}}+\mathrm{h} \rho_{\mathrm{w}} \mathrm{g}+\text { Asin } \omega \mathrm{t} \\
& \mathrm{P}_{\mathrm{g}}=\mathrm{P}_{\mathrm{vap}, \mathrm{PFC}}\left(\mathrm{T}_{\mathrm{s}, \mathrm{PFC}}\right)+\mathrm{P}_{\mathrm{vap}, \mathrm{H} 2 \mathrm{O}}\left(\mathrm{T}_{\mathrm{s}, \mathrm{H} 2 \mathrm{O}}\right) \\
& P_{O}=P_{g}-\frac{2 \gamma}{R(t)}
\end{aligned}
$$

The vapor pressures can be obtained from look-up tables, or from thermodynamic correlation equations. For example, the vapor pressures of $\mathrm{PFC}_{5}, \mathrm{PFC}_{6}$ and water can be obtained from empirical equations in the form of $\mathrm{Pvap}(\mathrm{Ts})=\exp \left(\mathrm{A}+\mathrm{B} / \mathrm{T}_{\mathrm{s}}+\mathrm{ClnT}_{\mathrm{s}}+\mathrm{DT}_{\mathrm{s}} \mathrm{E}_{\mathrm{s}}\right.$, where A, B, C, D and E are constants [42]. The coefficients used in this study are listed in Table 1.

Consider a spherical shell of gas of radius R, consisting of only PFC and water vapor, symmetrically surrounding a sphere of PFC liquid of radius $\mathrm{R}_{\mathrm{L}}$. The radial position within any phase is denoted by distance $r$ from the center of the nanodroplet, the pressure by $\mathrm{P}(\mathrm{r}, \mathrm{t})$, radial outward velocity of water by $u(r, t)$, and the temperature by $\mathrm{T}(\mathrm{r}, \mathrm{t})$.

The gas phase pressure, $\mathrm{P}_{\mathrm{g}}$, is a function of the liquid interface temperatures of the adjacent liquid phases. If these interfacial temperatures are dissimilar, then a thermal gradient will exist in the

Table 1

Vapor pressure coefficients of liquids used in this study [42].

\begin{tabular}{llllll}
\hline Compound & A & B & C & D & \\
\hline Water & $7.3649 \mathrm{E}+01$ & $7.2582 \mathrm{E}+03$ & $7.3037 \mathrm{E}+00$ & $4.1653 \mathrm{E} 06$ & $6.1648 \mathrm{E} 17$ \\
$\mathrm{PFC}_{5}$ & $7.2801 \mathrm{E}+01$ & $5.5390 \mathrm{E}+03$ & $7.5271 \mathrm{E}+00$ & $6.0000 \mathrm{E}+00$ \\
$\mathrm{PFC}_{6}$ & $6.3705 \mathrm{E}+01$ & $5.6667 \mathrm{E}+03$ & $6.0445 \mathrm{E}+00$ & $2.7396 \mathrm{E} 17$ & $6.0000 \mathrm{E}+00$ \\
\hline
\end{tabular}

gas phase. An average temperature, $T_{g}$, will be defined by assuming a linear temperature gradient between the two surfaces and integrating over the spherical shell: 


$$
\begin{aligned}
\overline{T_{g}} & =\frac{\int_{R_{L}}^{R} 4 \pi r^{2} T(r) \rho_{g} \overline{C_{p} M_{w}} d r}{\int_{R_{L}}^{R} 4 \pi r^{2} \rho_{g} \overline{C_{p} M_{w}} d r} \\
& =T_{s, P F C}+\frac{3}{4} \frac{\left(T_{s, H_{2} O}-T_{S, P F C}\right)\left(R^{4}-R_{L}^{4}\right)}{\left(R-R_{L}\right)\left(R^{3}-R_{L}^{3}\right)}-\frac{\left(T_{S, H_{2} O}-T_{s, P F C}\right) R_{L}}{\left(R-R_{L}\right)}
\end{aligned}
$$

where $\rho_{g}, C_{p}$ and $M_{w}$ are the density, the average heat capacity and the average molecular weight of the gas phase. Since the gas phase is at fairly low pressure is given by the ideal gas law:

$$
\rho_{g}=\frac{P_{g} \overline{M_{w}}}{R_{G} \overline{T_{g}}}
$$

where $R_{G}$ is the ideal gas constant. $M_{w}$ is the mole-averaged molecular weight of the gas phase:

$$
\overline{M_{w}}=X_{P F C} M_{P F C}+\left(1-X_{P F C}\right) M_{\mathrm{H}_{2} \mathrm{O}},
$$

where $\mathrm{X}_{\mathrm{PFC}}$ is the mole fraction of $\mathrm{PFC}$ in the gas phase:

$$
X_{P F C}=\frac{P_{\text {vap }, P F C}}{P_{g}}=\frac{P_{\text {vap }, P F C}\left(T_{s, P F C}\right)}{P_{\text {vap }, P F C}\left(T_{s, P F C}\right)+P_{\text {vap }, \mathrm{H}_{2} \mathrm{O}}\left(T_{s, \mathrm{H}_{2} \mathrm{O}}\right)} .
$$

Because the numerator and denominator of Eq. (7) change similarly with temperature, $\mathrm{X}_{\mathrm{PFC}}$ is a very weak function of temperature, and thus $d M w / d T g$ and $d X_{p r c} / d T_{g}$ are assumed to be zero in this simplified model. Furthermore, this means that the ratio of the rates of evaporation of PFC and water remains fairly constant for the small thermal changes that occur during evaporative cooling:

$$
\frac{d m_{P F C}}{d m_{H 2 O}}=\frac{X_{P F C} M_{P F C}}{\left(1-X_{P F C}\right) M_{H 2 O}}
$$

where $\mathrm{m}_{\mathrm{PFC}}$ and $\mathrm{m}_{\mathrm{H} 2} \mathrm{O}$ represents the masses of the liquid phases.

Because the vapor pressures change only slowly with average gas temperature, the change in gas density with respect to $T_{g}$ and $t$ can be approximated by

$$
\frac{d \rho_{g}}{d \overline{T_{g}}}=-\frac{P_{g} \overline{M_{w}}}{R_{G} \bar{T}_{g}^{2}}
$$

and,

$$
\frac{d \rho_{g}}{d t}=\left(\frac{-P_{g} \overline{M_{w}}}{R_{G} \bar{T}^{2}}\right) \frac{d \overline{T_{g}}}{d t}
$$


By conservation of mass in the incompressible water phase,

$$
u(r, t)=\frac{F(t)}{r^{2}}, \quad \text { for } r \geqslant R
$$

where $F(t)$ is some function related to $R(t)$ by a kinematic boundary condition at the bubble surface. Neglecting the very little evaporation of water from the gas/water interface, $u(R, t)=d R / d t$, and therefore

$$
F(t)=R^{2} \frac{d R}{d t}
$$

This case is appropriate for our model in which the vapor pressure PFC is much larger than that of water, and the density of liquid water $\left(1000 \mathrm{~kg} / \mathrm{m}^{3}\right)$ is much greater than the gas density $\left(<0.34 \mathrm{~kg} / \mathrm{m}^{3}\right)$.

The mass rate of evaporations of PFC liquid and water into the gas phase produce a change in the mass of the gas phase, $\mathrm{m}_{\mathrm{g}}$, given by:

$$
\begin{aligned}
-\frac{d m_{P F C}}{d t}-\frac{d m_{\mathrm{H}_{2} \mathrm{O}}}{d t}=\frac{d m_{g}}{d t}=\frac{d\left(V_{g} \rho_{g}\right)}{d t}=\rho_{g} & {\left[4 \pi R^{2} \frac{d R}{d t}-4 \pi R_{L}^{2} \frac{d R_{L}}{d t}\right] } \\
& +\frac{4}{3} \pi\left(R^{3}-R_{L}^{3}\right) \frac{d \rho_{g}}{d t} .
\end{aligned}
$$

The left side of Eq. (13) can be combined with Eq. (8) to relate the gas phase gain to the PFC liquid loss:

$$
\frac{d m_{g}}{d t}=-\frac{d m_{P F C}}{d t}-\frac{d m_{\mathrm{H}_{2} \mathrm{O}}}{d t}=-\frac{d m_{P F C}}{d t}\left[1+\frac{\left(1-X_{P F C}\right) M_{\mathrm{H}_{2} \mathrm{O}}}{X_{P F C} M_{P F C}}\right] .
$$

The rate of PFC evaporation is related to the change in liquid droplet diameter by:

$$
-\frac{d m_{P F C}}{d t}=-\rho_{P F C} 4 \pi R_{L}^{2} \frac{d R_{L}}{d t}=M_{P F C} \frac{d\left(X_{P F C} N\right)}{d t} .
$$

where $\mathrm{N}$ is the total number of moles in the gas phase.

Finally, we desire to connect all of these changes $\left(R, R_{L}, q_{g}, T_{g}\right.$, etc.) to a single parameter that we can track with time, namely the change in the moles of gas phase, $\underline{\mathrm{dN}}_{\mathrm{dt}}$. Combination with Eq. (13) gives: 


$$
\begin{aligned}
\frac{d N}{d t} & =\frac{1}{\overline{M_{w}}} \frac{d m_{g}}{d t} \\
& =\frac{4 \pi \rho_{g}}{\overline{M_{w}}}\left[R^{2} \frac{d R}{d t}-R_{L}^{2} \frac{d R_{L}}{d t}\right]+\frac{4 \pi}{3 \overline{M_{w}}}\left(R^{3}-R_{L}^{3}\right) \frac{d \rho_{g}}{d t} .
\end{aligned}
$$

Substituting Eqs. (10), (14), and (15) into (16) produces a relationship between three key time derivatives of R, N, and $\mathrm{T}_{\mathrm{g}}$ :

$$
\begin{gathered}
\rho_{g} 4 \pi R^{2} \frac{d R}{d t}-\frac{4}{3} \pi\left(R^{3}-R_{L}^{3}\right)\left(\frac{P_{g} \overline{M_{w}}}{R_{G} \overline{T_{g}}}\right) \frac{d \overline{T_{g}}}{d t} \\
=\left[\overline{M_{w}}-\frac{\rho_{g}}{\rho_{P F C}} X_{P F C} M_{P F C}\right] \frac{d N}{d t} .
\end{gathered}
$$

The term on the right in brackets looks very much like a modified molecular weight. In fact, if we consider that the gas density $\left(3.95 \mathrm{~kg} / \mathrm{m}^{3}\right.$ at 25$)$ is much less that the liquid PFC density $\left(1670 \mathrm{~kg} / \mathrm{m}^{3}\right.$ at 25$)$, the term with the ratio of densities will be negligible, leaving only the mole-averaged molecular weight term.

From Eq. (17) the velocity of the water interface at the bubble surface is given by

$$
u(R, t)=\frac{d R}{d t}=\frac{R_{G} \overline{T_{g}}}{4 \pi R^{2} P_{g}} \frac{d N}{d t}+\frac{\left(R^{3}-R_{L}^{3}\right)}{3 \overline{T_{g}} R^{2}} \frac{d \overline{T_{g}}}{d t} .
$$

Thus from Eqs. (11), (12), and (18) the velocity in the liquid water phase (for $r>=\mathrm{R}$ ) is given by:

$$
u(r, t)=\frac{R_{G} \overline{T_{g}}}{4 \pi r^{2} P_{g}} \frac{d N}{d t}+\frac{\left(R^{3}-R_{L}^{3}\right)}{3 r^{2}} \frac{d \ln \overline{T_{g}}}{d t}
$$

and

$$
F(t)=R^{2} \frac{d R}{d t}=\frac{R_{G} \overline{T_{g}}}{4 \pi P_{g}} \frac{d N}{d t}+\frac{\left(R^{3}-R_{L}^{3}\right)}{3} \frac{d \ln \overline{T_{g}}}{d t}
$$

The momentum balance is derived by applying the Navier- Stokes equations [50] to the previous mass balance to obtain a velocity distribution in the water phase. First, Eq. (19) is differentiated with respect to $\mathrm{r}$ : 


$$
\frac{\partial u(r, t)}{\partial r}=-\frac{R_{G} \overline{T_{g}}}{2 \pi r^{3} P_{g}} \frac{d N}{d t}-\frac{2}{3 r^{3}}\left(R^{3}-R_{L}^{3}\right) \frac{d \ln \overline{T_{g}}}{d t} .
$$

Then the acceleration of the gas/water interface is given by:

$$
\frac{\partial u(r, t)}{\partial t}=\frac{R_{G} \overline{T_{g}}}{4 \pi r^{2} P_{g}} \frac{d^{2} N}{d t^{2}}+\frac{1}{3 r^{2}}\left(R^{3}-R_{L}^{3}\right) \frac{d^{2} \ln \overline{T_{g}}}{d t^{2}} .
$$

The Navier-Stokes equation in spherical coordinates for water motion in the $\mathrm{r}$ direction is:

$$
-\frac{1}{\rho_{L}} \frac{\partial P}{\partial r}=\frac{\partial u}{\partial t}+u \frac{\partial u}{\partial r}-v_{L}\left[\frac{1}{r^{2}} \frac{\partial^{2}}{\partial r^{2}}\left(r^{2} u\right)\right],
$$

for $r>=R$, where $V_{L}$ is the kinematic viscosity of the water phase.

Combination of Eqs. (19), (21), (22), and (23) produces:

$$
\begin{aligned}
-\frac{1}{\rho_{L}} \frac{\partial P}{\partial r}= & \frac{1}{r^{2}}\left[\frac{R_{G} \overline{T_{g}}}{4 \pi P_{g}} \frac{d^{2} N}{d t^{2}}+\frac{1}{3}\left(R^{3}-R_{L}^{3}\right) \frac{d^{2} \ln \overline{T_{g}}}{d t^{2}}\right] \\
& -\frac{2}{r^{5}}\left[\frac{R_{G}^{2} \overline{T_{g}}}{16 \pi^{2} P_{g}^{2}}\left(\frac{d N}{d t}\right)^{2}+\frac{R_{G} \overline{T_{g}}\left(R^{3}-R_{L}^{3}\right)}{6 \pi P_{g}} \frac{d N}{d t} \frac{d \ln \overline{T_{g}}}{d t}+\frac{\left(R^{3}-R_{L}^{3}\right)^{2}}{9}\left(\frac{d \ln \overline{T_{g}}}{d t}\right)^{2}\right],
\end{aligned}
$$

in which the term in brackets of Eq. (23) vanishes.

Combination of Eqs. (20) and (24) becomes:

$$
-\frac{1}{\rho_{L}} \frac{\partial P}{\partial r}=\frac{1}{r^{2}} \frac{\partial F}{\partial t}-\frac{2 F^{2}}{r^{5}}
$$

Integration of Eq. (25) with respect to $\mathrm{r}$ between the limits of $\mathrm{P}=\mathrm{P}_{\mathrm{o}}$ at $\mathrm{r}=\mathrm{R}$, and $\mathrm{P}=\mathrm{P}_{\infty}$ at $\mathrm{r}=\infty$ produces:

$$
\frac{P_{o}-P_{\infty}}{\rho_{L}}=\frac{1}{R} \frac{d F}{d t}-\frac{1}{2} \frac{F^{2}}{R^{4}}
$$


where $P_{o}$ is the pressure in the water just outside the gas/water interface.

To consider the dynamic boundary condition on the bubble surface, a small thin lamina containing a segment of the gas/water interface is shown in Fig. 3. The net force per unit area $(\mathrm{F} / \mathrm{A})_{\text {net }}$ on this lamina in the radially outward direction per unit area is

$$
(\mathscr{F} / A)_{n e t}=\left.\pi_{r r}\right|_{r=R}+P_{g}-2 \gamma / R
$$

where $p_{\mathrm{rr}}$ is the radial component of the molecular stress tensor in the outward direction. This component is given by $\pi_{\Pi}=$ $-P_{o}+2 \mu_{L} \partial u / \partial r$

Therefore, the force per unit area is:

$$
(\mathscr{F} / A)_{n e t}=-P_{o}+2 \mu_{L} \frac{\partial u}{\partial r}+P_{g}-2 \gamma / R
$$

By combining Eqs. (19) and (21),

$$
\left(\frac{\partial u}{\partial r}\right)_{\mid r=R}=-\frac{2}{R} u(R, t)=-\frac{2}{R} \frac{d R}{d t}
$$

Substituting Eq. (29) into Eq. (28) gives:

$$
(\mathscr{F} / A)_{n e t}=-P_{o}-\frac{4 \mu_{L}}{R} \frac{d R}{d t}+P_{g}-2 \gamma / R
$$

In the absence of mass transport across the gas/water boundary, this force per unit area must be equal to zero:

$$
0=-P_{o}-\frac{4 \mu_{L}}{R} \frac{d R}{d t}+P_{g}-2 \gamma / R
$$




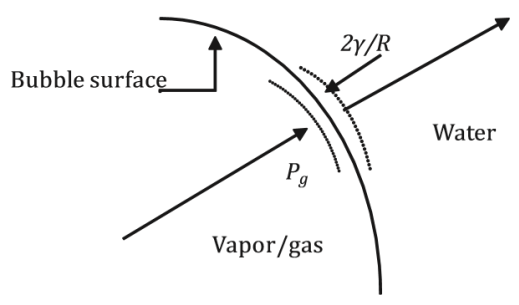

Fig. 3. Force balance on bubble/water interface.

Substitution Eqs. (26) and (20) into Eq. (31), we obtain the Pitt modification of the Rayleigh-Plesset equation:

$$
\frac{P_{g}(T, t)-P_{\infty}(t)}{\rho_{L}}=R \frac{d^{2} R}{d t^{2}}+\frac{3}{2}\left(\frac{d R}{d t}\right)^{2}+\frac{4 v_{L}}{R} \frac{d R}{d t}+\frac{2 \gamma}{R \rho_{L}}
$$

The difference between this and the classical Rayleigh-Plesset equation is that the internal bubble pressure, $\mathrm{P}_{\mathrm{g}}$, is a function of temperature, not of bubble size, because in the 2-phase volume inside the gas-water boundary, the vapor pressures of the PFC and water determine the internal gas-phase pressure.

This second order ordinary differential equation can be solved using numerical techniques. In this research we used MATLAB software (MathWorks, Inc., Natick, MA) and employed the ODE45 subroutine to obtain the functions $\mathrm{R}(\mathrm{t}), \mathrm{dR}(\mathrm{t}) / \mathrm{dt}$, $\mathrm{dN}(\mathrm{t}) / \mathrm{dt}$ and $\mathrm{dTg} / \mathrm{dt}$. ODE45 is an explicit Runga-Kutta routine that uses a fourth order approximation to calculate and a fifth order to truncate the solution.

Although some applications of ultrasound are used to heat tissues, ultrasonic energy is absorbed very little by pure water [51]. On the time scales used in this model, any thermal effects due to absorption of ultrasonic energy in the gas or liquid phases are negligible. When ultrasound nucleates a PFC gas bubble, evaporation from the PFC liquid surface decreases its surface temperature, thus creating a temperature gradient in the adjacent gas and liquid phases. Similarly, the small amount of evaporation from the gas/ water interface will also create cooling and thermal gradients. Because the pressure and composition in the gas phase is a function of the interface temperatures, we must perform an energy balance to track these temperatures during expansion and contraction of the gas phase.

The governing equation for the temperature distribution in the PCF droplet is given by:

$$
\rho_{P F C} C_{P, P F C} \frac{d T}{d t}=\frac{-1}{r^{2}} \frac{\partial}{\partial r}\left(r^{2} q_{i n t, P F C}\right), \quad \text { for } r<R_{L},
$$

where $\mathrm{C}_{\mathrm{P} ; \mathrm{PFC}}$ is the heat capacity of the PFC liquid, and $\mathrm{q}_{\mathrm{int} ; \mathrm{PFC}}$ is the energy flux within the PFC liquid droplet. This equation is subject to an energy flux boundary condition at $r=R_{L}$, at which the interfacial heat flux at the surface, $\mathrm{q}_{\mathrm{s} ; \mathrm{PFC}}$, equals the flux on the PFC side of the interface ( $\left.\mathrm{q}_{\mathrm{int}} \mathrm{PFC}\right)$ and also equals the flux out from the surface on the gas-phase side:

$$
q_{\text {int }, \text { PFC }}=q_{s, P F C}=q_{\text {c.gas }}+q_{\text {rad }}+q_{\text {evap }} .
$$


In this equation, $\mathrm{q}_{\mathrm{s} ; \mathrm{PFC}}$ is the net energy flux at the surface of the PCF phase, $\mathrm{q}_{\mathrm{c} ; \mathrm{gas}}$ is the heat transferred from the interface by conduction through the gas phase, $\mathrm{q}_{\mathrm{rad}}$ is the heat transferred from the interface by radiation through the gas phase, and $\mathrm{q}_{\mathrm{e}} \mathrm{ap}$ is the latent heat transferred away from the liquid interface by the phase change (this will be a positive quantity during evaporation). These fluxes in turn are given by:

$$
\begin{aligned}
& q_{s, P F C}=-\left.k_{P F C} \frac{\partial T}{\partial r}\right|_{r=R_{L}} \\
& q_{c, \text { gas }}=-\left.k_{\text {gas }} \frac{\partial T}{\partial r}\right|_{r>R_{L}} \cong-\theta h\left(T_{s, H_{2} O}-T_{s, P F C}\right) \\
& q_{\text {rad }}=-\varepsilon \sigma f\left(T_{s, H_{2} O}^{4}-T_{s, P F C}^{4}\right) \\
& q_{\text {evap }}=\frac{M_{P F C} \Delta H_{P F C}}{4 \pi R_{L}^{2}} \frac{d N_{P F C}}{d t}=\frac{M_{P F C} \Delta H_{P F C} X_{P F C}}{4 \pi R_{L}^{2}} \frac{d N}{d t}
\end{aligned}
$$

where $\mathrm{k}_{\mathrm{gas}}$ is the average thermal conductivity of the gas phase, $\mathrm{h}$ is the local heat transfer coefficient at the interface, $\mathrm{h}$ is the blowing factor, $\mathrm{e}$ is the emissivity of the interface, $\mathrm{r}$ is the Stefan-Boltzmann constant, $\mathrm{f}$ is the view factor at the PFC interface, and $\mathrm{DH}_{\mathrm{PFC}}$ and $\mathrm{k}_{\mathrm{PFC}}$ are the enthalpy of vaporization and thermal conductivity of the PFC liquid, respectively. The right-most term in Eq. (36) is only approximate because the actual thermal profile may not be linear, but the deviation from linearity will be considered negligible in the following calculations. For a sphere with no transverse convection, the Nusselt number is given by $\mathrm{Nu}$ $1 / 421 / 4 \mathrm{hD}=\mathrm{k}$ [50]. Therefore in Eq. (36), $\mathrm{h}=\mathrm{k}_{\mathrm{gas}} / \mathrm{R}_{\mathrm{L}}$. The blowing factor corrects for the high mass transfer at the evaporating (or condensing) interface [50]. For our model,

$$
\begin{aligned}
& \theta=\frac{\phi}{e^{\phi}-1}, \\
& \phi=\frac{X_{P F C} C_{P, P F C}}{4 \pi R_{L} k_{g a s}} \frac{d N}{d t}
\end{aligned}
$$

The view factor, $f$, from the surface of the PFC droplet to the surrounding water surface is unity.

Eqs. (36)-(40) are easily included into the Runga-Kutta solution described above because $\mathrm{dN}=\mathrm{dt}$ is readily calculated by mass balance. However, Eq. (35) is problematic to employ in a Runga-Kutta routine because the thermal distribution within the droplet is not easily calculated in one step. Thus $\mathrm{T}_{\mathrm{S}}$ cannot be determined exactly. However, we can "bracket" the true result with approximate solutions for $\mathrm{T}_{\mathrm{s}}$. At one extreme, we can assume that the PFC thermal diffusivity is high enough, the spatial distance short enough, and the time scale long enough that the droplet remains in thermal equilibrium with no internal temperature gradients; and thus $\mathrm{T}_{\mathrm{s}}=\mathrm{T}_{\text {droplet. }}$. This case corresponds to the Fourier number approaching infinity, where the Fourier number is defined as 
$F_{o}=\frac{\alpha t}{R_{L}^{2}}$ and thermal diffusivity as $\alpha=\mathrm{K}_{\mathrm{PFC}} / \rho \mathrm{C}_{\mathrm{p}, \mathrm{PFC}}$ The change in droplet temperature is the interfacial flux divided by the heat capacity and mass of the droplet, giving:

$$
\begin{aligned}
\frac{d T_{\text {droplet }}}{d t} & =\frac{d T_{s, P F C}}{d t} \\
& =\frac{3}{\rho C_{P, P F C} R_{L}}\left[\theta h\left(T_{s, H_{2} O}-T_{s, P F C}\right)+\varepsilon \sigma f\left(T_{s, H_{2} O}^{4}-T_{s, P F C}^{4}\right)-\frac{M_{P F C} \Delta H_{P F C} X_{P F C}}{4 \pi R_{L}^{2}} \frac{d N}{d t}\right]
\end{aligned}
$$

Since the PFC droplet always has a spatially uniform temperature, we call this the "uniform temperature model".

Substitution of $\underline{\mathrm{dN}}_{\mathrm{dt}}$ from Eqs. (17) and (34) into Eq. (41) relates the PFC surface temperature to $\mathrm{dR} / \mathrm{dt}$, which in turn is driven by the dynamic pressure in the Raleigh-Plessett Equation.

The other extreme of the "bracket" condition for $\mathrm{T}_{\mathrm{s}}$ is a situation in which the thermal diffusion length is small enough, the droplet radius large enough, and the time short enough such that the droplet appears to be a semi-infinite volume with a thermal change imposed at the boundary that moves into the PFC liquid droplet with time. This model pertains to a classical solution called the "penetration model".

To include this in the Runga-Kutta solution, we must get a function for $\mathrm{dT}_{\mathrm{s}} / \mathrm{dt}$. It is observed that the thermal penetration distance into a semi-infinite phase (in a rectangular coordinate system) with constant surface temperature increases proportional to the square root of time. Likewise, in the case of a constant thermal flux into a semi-infinite phase, the change in surface temperature changes proportional to the square root of time:

$$
T_{s}(t)-T_{o}=\frac{q_{o}}{k} \sqrt{\frac{4 \alpha t}{\pi}}
$$

where $T_{0}$ and $k$ are the initial temperature and thermal conductivity of the semi-infinite phase (the PFC liquid droplet), and $q_{o}$ is the applied flux at the interface. However, in our model of the evaporation of PFC from the droplet surface, the flux will not be constant throughout the entire evaporation and condensation cycle, and thus the use of Eq. (42) is not rigorously accurate. However, the use of Eq. (42) with a varying $q_{o}$ is still a good estimate because it captures the key feature of thermal penetration - that the change in temperature is proportional to the root of time during the application of the thermal flux. Differentiation of Eq. (42) gives

$$
\frac{d T_{s, P F C}}{d T}=\frac{q_{s, P F C}}{k_{P F C}} \sqrt{\frac{\alpha}{\pi\left(t-t^{\prime}\right)}}
$$

where $t^{0}$ is the time of the first appearance of the gas phase and $\mathrm{q}_{\mathrm{s} ; \mathrm{PFC}}$ is defined in Eqs. (34)-(40). 


\section{Results}

\subsection{Comparison of thermal models}

Using mass, momentum and energy balances, we derived two slightly different models for gas bubble behavior - one with a thermal boundary layer in the PFC liquid nanodroplet (penetration model), and one without any thermal boundary layer (uniform temperature model). These models produce two slightly different temperatures for the liquid surface. The actual temperature (and true behavior of the gas phase) lies between these two bounding models. Fig. 4 shows a typical output from the numerical simulation of each model. In this figure, a $250-\mathrm{nm}$ diameter emulsion droplet of $\mathrm{PFC}_{6}$ at $25 \mathrm{C}$ was subjected to $500-\mathrm{kHz}$ insonation at $99 \mathrm{kPa}$ amplitude with $20 \mathrm{~mm}$ hydrostatic pressure (196 Pa). In this and some subsequent figures, the top panel shows the local pressure variation due to insonation; the second panel shows the bubble radius; the third panel shows the bubble surface velocity; the fourth panel shows the emulsion droplet surface temperature.

The first thing to note is that the gas phase does not start forming until the pressure inside the PFC droplet (local pressure plus Laplace pressure) decreases to below the vapor pressure of the $\mathrm{PFC} / \mathrm{H}_{2} \mathrm{O}$ combination. The bubble radius increases to nearly twice the size of the emulsion droplet, and then shrinks quickly with a collapse velocity approaching $2.5 \mathrm{~m} / \mathrm{s}$.

The fourth panel shows the surface temperature predicted by the 2 bounding models. There are slight differences; the surface in the penetration model did not cool or reheat as much as the uniform temperature model. However, these small differences do not produce significant difference in the bubble dynamics, as the middle panels show near perfect superposition of the outputs from the two models. Since both bracketing extremes produce similar bubble wall dynamics, most of the subsequent figures show only results from the uniform temperature model. One curious outcome of the model is that the PFC droplet surface temperature heats up to exceed its initial temperature during the gas phase condensation. To determine where this "excess" energy comes from, we did several numerical experiments in which the various heat transfer terms of Eq. (34) were eliminated. The solid line of Fig. 5 shows the thermal dynamics when the conductive heat transfer term (Eq. (36)) was eliminated; the temperature at the end of PFC condensation was the same as the initial temperature. This result shows that during the very short time that the PFC droplet surface is cooler than the surrounding water temperature, there is significant heat transfer across the gaseous gap between water and PFC liquid. This heating continues as long as the droplet surface is cooler than the water; then the heat flow reverses direction during the last stages of condensation when the droplet surface is warmer than the water, producing a downward curving profile during the very end of gas phase collapse. Once the gas phase is gone, the PFC liquid continues to cool by conduction to the water. The numerical experiments also showed that radiative heat transfer is negligible during gas expansion and condensation.

Fig. 6 shows a comparison of $\mathrm{PFC}_{6}$ and $\mathrm{PFC}_{5}$ emulsions at the same temperature $(25 \mathrm{C})$ and frequency $(500 \mathrm{kHz})$ but different pressure amplitudes, selected such that the gas phase was initiated at the same time in the acoustic cycle. The maximum bubble radius is larger in $\mathrm{PFC}_{6}$ because the acoustic pressure is higher. The collapse velocity is also greater. Both bubbles continue to grow larger, long past the point of greatest rarefaction pressure (most negative pressure) due to the outward momentum of the moving water. Although the bubble growth started with both liquids at the same time, the growth of $\mathrm{PFC}_{6}$ bubble was greater because the subpressurization was greater due to the higher amplitude imposed on the $\mathrm{PFC}_{6}$. This larger bubble size led to a much greater collapse velocity and the bubble was terminated sooner than the $\mathrm{PFC}_{5}$ bubble that had only $1 / 3$ of the collapse velocity of the $\mathrm{PFC}_{6}$. The dynamic model reveals some very interesting details that had not been expected a priori. 


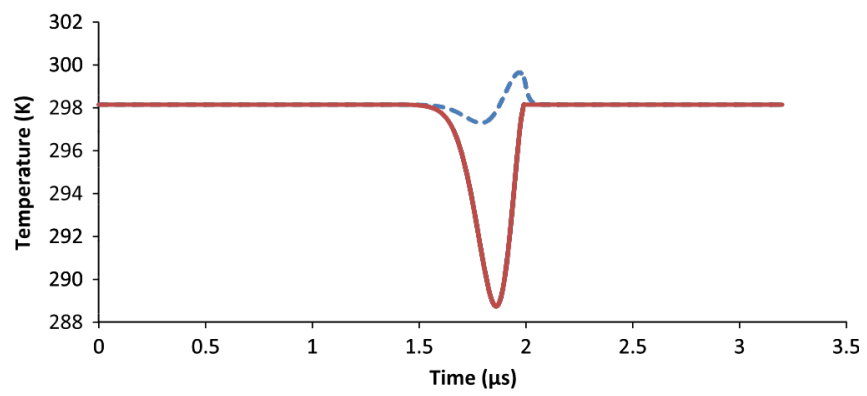

Fig. 5. Effect of conductive heat transfer. A 250-nm diameter emulsion droplet of $\mathrm{PFC}_{6}$ at $25 \mathrm{C}$ was subjected to $500-\mathrm{kHz} \mathrm{US}$ at $115 \mathrm{kPa}$ amplitude using the uniform temperature model. The dashed line indicates the surface temperature produced by the cumulative effect of conduction, evaporation, and radiation heat transfer. The solid line neglects the contribution of conduction, but still retains radiation heat transfer, which is negligible.

\subsection{Effect of acoustic amplitude on bubble dynamics at $500 \mathrm{kHz}$}

When a $250-\mathrm{nm} \mathrm{PFC}_{6}$ emulsion is exposed to $500-\mathrm{kHz}$ US at different acoustic amplitudes, the maximum radius of the gas phase increases with the acoustic amplitude, as can be seen on the Fig. 6. The bubble radius grows to $350 \mathrm{~nm}$ at an acoustic amplitude of $99 \mathrm{kPa}$. The same general trend is observed with other sizes and with the $\mathrm{PFC}_{5}$ emulsions. The smaller amplitudes produce earlier
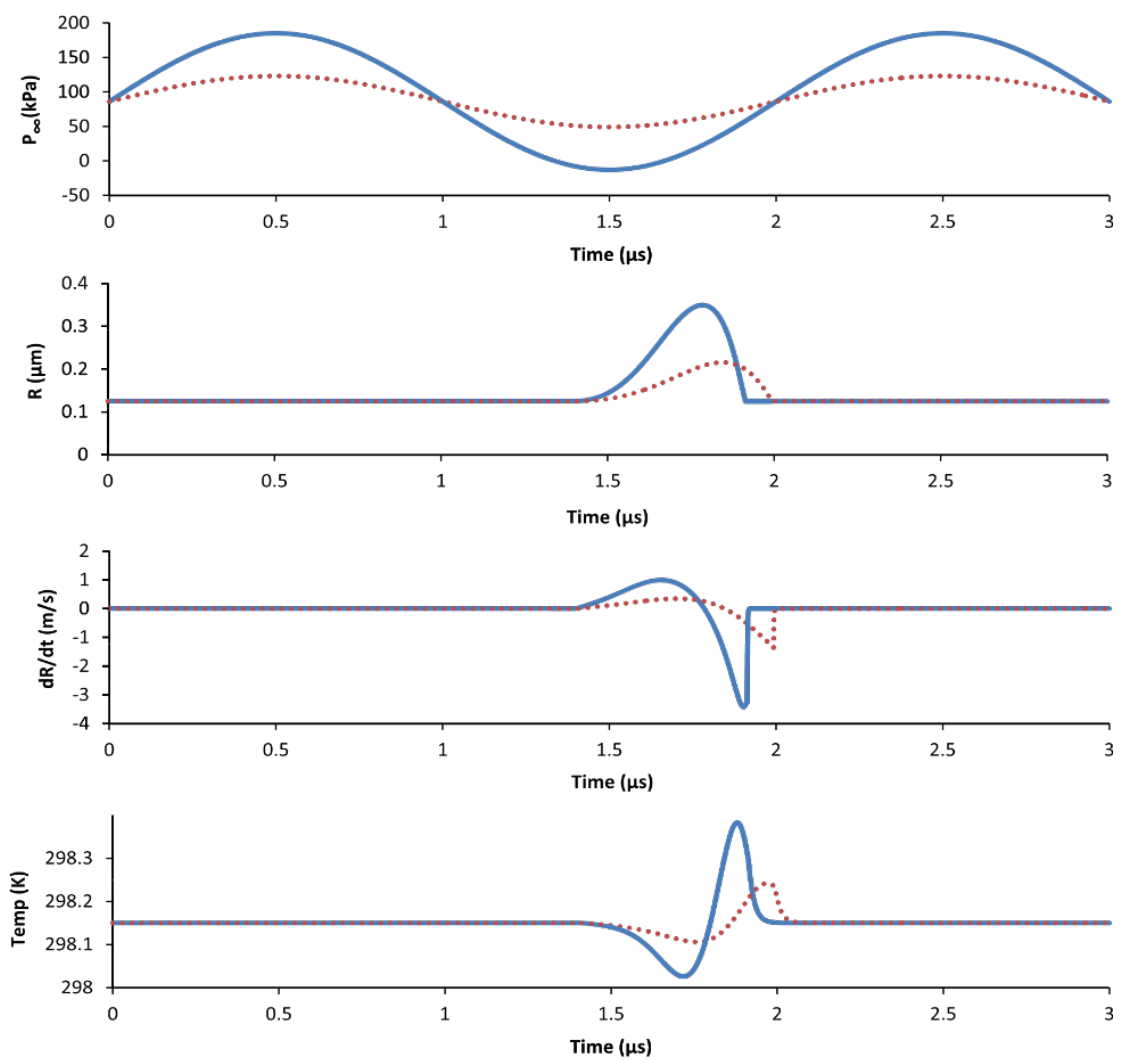

Fig. 6. Comparison of $\mathrm{PFC}_{6}$ and $\mathrm{PFC}_{5}$. In this figure, a 250-nm diameter emulsion droplet at $25 \mathrm{C}$ was subjected to $500-\mathrm{kHz}$ US. The solid line signifies $\mathrm{PFC}_{6}$ and the dotted line signifies $\mathrm{PFC}_{5}$. The emulsion droplets were subjected to $99 \mathrm{kPa}$ and $37 \mathrm{kPa}$, respectively, so that they commenced gas formation at a similar time. Surface tension is $2.5 \mathrm{mN} / \mathrm{m}$. 
collapse because there is less time available for growth. In Fig. 6, at acoustics amplitudes of $94 \mathrm{kPa}$ and lower, there was no initiation of bubble growth.

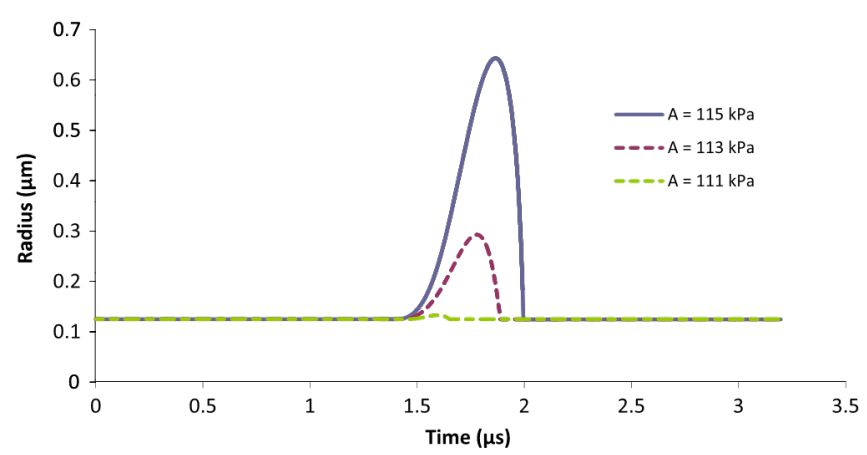

Fig. 7. Effect of acoustic amplitude on bubble radius. 250-nm diameter $\mathrm{PFC}_{6}$ emulsion exposed to varying acoustic amplitudes at 500-kHz US and $25 \mathrm{C}$. The surface tension was $3.5 \mathrm{mN} / \mathrm{m}$.

Fig. 7 shows the maximum radius of the gas bubble, which increases greatly with acoustic amplitude once the gas formation threshold, has been surpassed (110.5 kPa). At an acoustic amplitude of $116 \mathrm{kPa}$, the liquid droplet is completely evaporated, but the bubble continues to expand due to outward expansion momentum. Thus there is a fairly small amplitude window in which only partial evaporation of the droplet occurs. This window is even narrower as the frequency is decreased, as will be shown later. Such narrow windows suggest that in real applications of acoustic droplet vaporization, the droplet will either not expand, or will expand completely to gas with a large volume increase. Our model assumed that the gas from a totally evaporated droplet would condense into a symmetrical concentric liquid droplet once the internal pressure exceeded the vapor pressure. However, in real situations, nucleation of the liquid phase may not occur immediately, and probably would not be concentric, thus leading to an unstable collapse and perhaps fragmentation of the droplet. The boundary layer model was used to examine the effect of acoustic amplitude on the maximum bubble radius and collapse velocity when 250- and 550-nm PFC $_{6}$ emulsions were exposed to 500-kHz ultrasound. The results of expansions and collapse velocities at $25 \mathrm{C}$ are shown in Fig. 8. These data show that once the gas formation threshold is exceeded, the $R_{\max }$ and collapse velocities are fairly linear functions of acoustic pressure, at least in the ranges explored. It is interesting that the smaller sized droplet produces a steeper slope in the plots of Fig. 8, indicating that expansion is more sensitive to pressure in smaller droplets. We attribute this to the higher Laplace pressure of the smaller droplets, which in turn require higher amplitudes that create larger pressure driving forces for bubble expansion once the bubble forms. A similar trend was observed for $\mathrm{PFC}_{5}$. When $\mathrm{PFC}_{6}$ nanoemulsions of varying initial sizes are exposed to the same acoustic pressure $(111 \mathrm{kPa})$ at $500 \mathrm{kHz}$ and 25 $\mathrm{C}$, the smaller emulsion droplets do not grow as much as the larger nanodroplets (see Fig. 9). This same trend is also seen with other acoustic pressures, temperatures, and frequencies, as well as with $\mathrm{PFC}_{5}$. Again this is related to the Laplace pressure. The bubbles start growing at slightly different times, with the larger emulsions initiating growth first because larger emulsion droplets have less Laplace pressure restraining the initial gas formation. They also grow larger because, after this early initiation, there is even less Laplace pressure (even greater R) as the expansion progresses. 


\subsection{Effect of frequency on bubble dynamics near $500 \mathrm{kHz}$}

Fig. 10 shows the effect of frequency on bubble growth between 450 and $1000 \mathrm{kHz}$ for $\mathrm{PFC}_{6}$ of both sizes $(250$ and $550 \mathrm{~nm})$ at both temperatures $(25$ and $37 \mathrm{C}$ ). These simulations were done at different acoustic amplitudes so they could be presented on the same plot. As the temperature increases, the vapor pressure increases and thus the expansion starts earlier in the cycle and the bubble become much larger than at lower temperatures (data not shown). The most important aspect of Fig. 10 is that that near 500 $\mathrm{kHz}$, the maximum gas bubble radius and its collapse velocity decrease with increasing frequency. This observation can be attributed to the shorter time window in which the gas cavity can expand. In fact, as the frequency approaches $1 \mathrm{MHz}$, the 250-nm emulsion shows very little expansion. Some gas does form, as evidenced by the collapse velocity in Fig. 10b remaining above zero.The collapse velocities shown in Fig. 10 are relatively slow compared to the speed of collapse of non-condensable gas bubbles, which approach the speed of sound in water in some simulations [52,53]. Such a slow collapse in our present simulation may not produce the same intensity of shock wave as generated when collapse occurs at much higher velocities. For comparison, consider a general gas bubble of non-condensable gasses $\left(\mathrm{O}_{2}, \mathrm{~N}_{2}\right.$, etc. $)$.

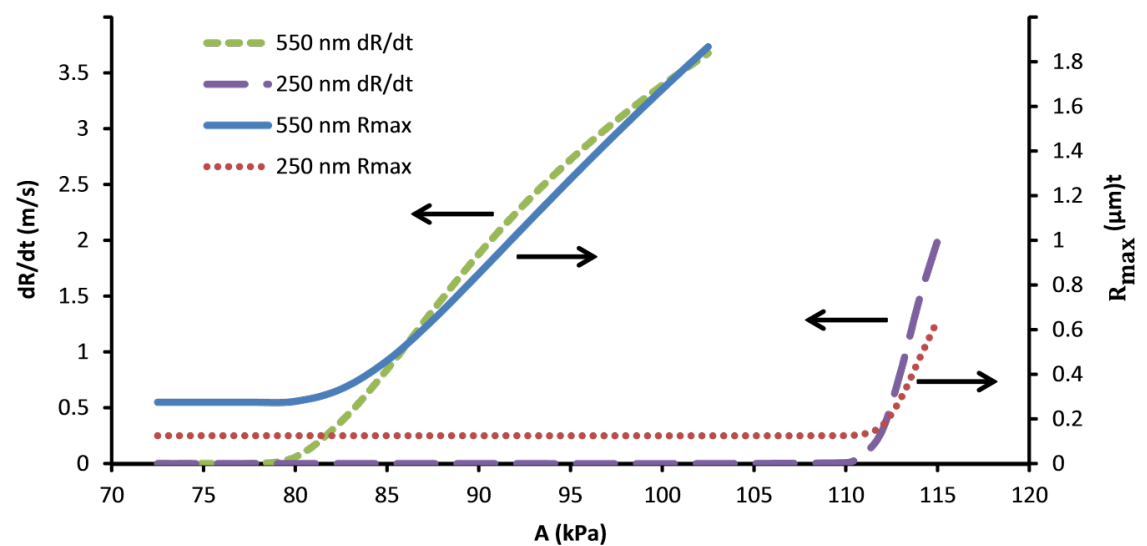

Fig. 8. Effect of acoustic amplitude on maximum collapse velocity (left axis) and maximum radius (right axis) of a $\mathrm{PFC}_{6}$ emulsion droplets. $\mathrm{The}^{250}$-nm diameter and 550-nm emulsion droplet at $25 \mathrm{C}$ were exposed to varying amplitudes at 500-kHz US.

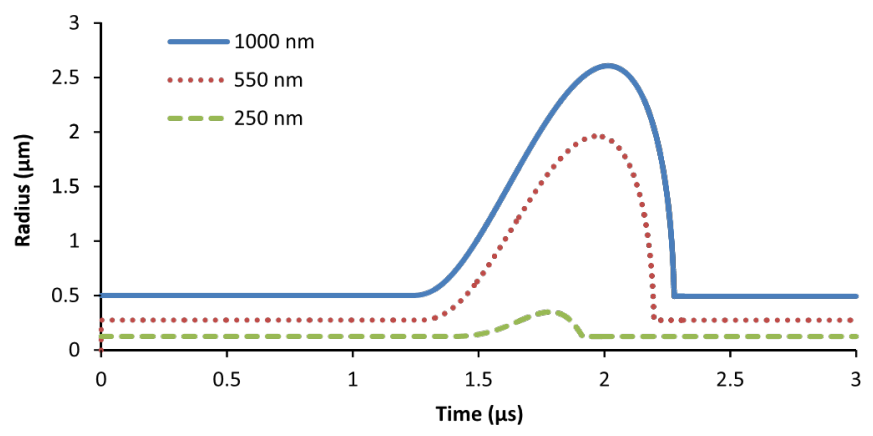

Fig. 9. Bubble radius of 250, 550 and $1000 \mathrm{~nm} \mathrm{PFC}_{6}$ emulsion droplets at $25 \mathrm{C}$ subjected to 500-kHz US at $111 \mathrm{kPa}$ amplitude, respectively.

During the rapid cavitational collapse of non-condensable gas bubbles, a spherical shell of a shock wave forms in the gas phase in front of the wall of water moving inward near the speed of sound. The implosion compresses the non-condensable gasses to a supercritical fluid of very high temperature and pressure, which in turn produces a shock wave that emanates from the implosion into the surrounding media. 
In our model, all of the gas is condensable perfluorocarbon or water, so no extreme temperatures are generated as these condense during bubble contraction. However, the sudden meeting of the collapsing wall of water and the perfluorocarbon liquid core will create an expanding pressure wave that should be experimentally detectable with a hydrophone. The conversion between fluid kinetic energy and pressure suggests that the magnitude of the pressure wave generated when the water wall slams into the perfluorocarbon core is on the order of $\mathrm{p}^{1 / 4} \mathrm{q}_{2} \mathrm{~V}_{\mathrm{t}}^{2}$, where $\mathrm{p}$ is the shock wave pressure, $\mathrm{q}$ is the water density, and $\mathrm{V}_{\mathrm{t}}$ is the terminal water velocity at impact. Thus the terminal velocity of a slow collapse of $4 \mathrm{~m} / \mathrm{s}$ would produce a pressure pulse on the order of 8 $\mathrm{kPa}$, or $0.08 \mathrm{~atm}$, which should be experimentally detectable with a hydrophone [48]. However, a faster collapse of $200 \mathrm{~m} / \mathrm{s}$ would produce a shock wave of about $20,000 \mathrm{kPa}(200 \mathrm{~atm})$.

\subsection{Effect of surface tension and temperature on bubble dynamics}

The surfactant acts as a stabilizing agent at the emulsion-water interface to keep the emulsion droplets from coalescing. For example, a charged surfactant creates electrostatic repulsion to preclude coalescence. Surfactants also decrease the surface tension to very low values so the Laplace pressure is not so excessive that gas
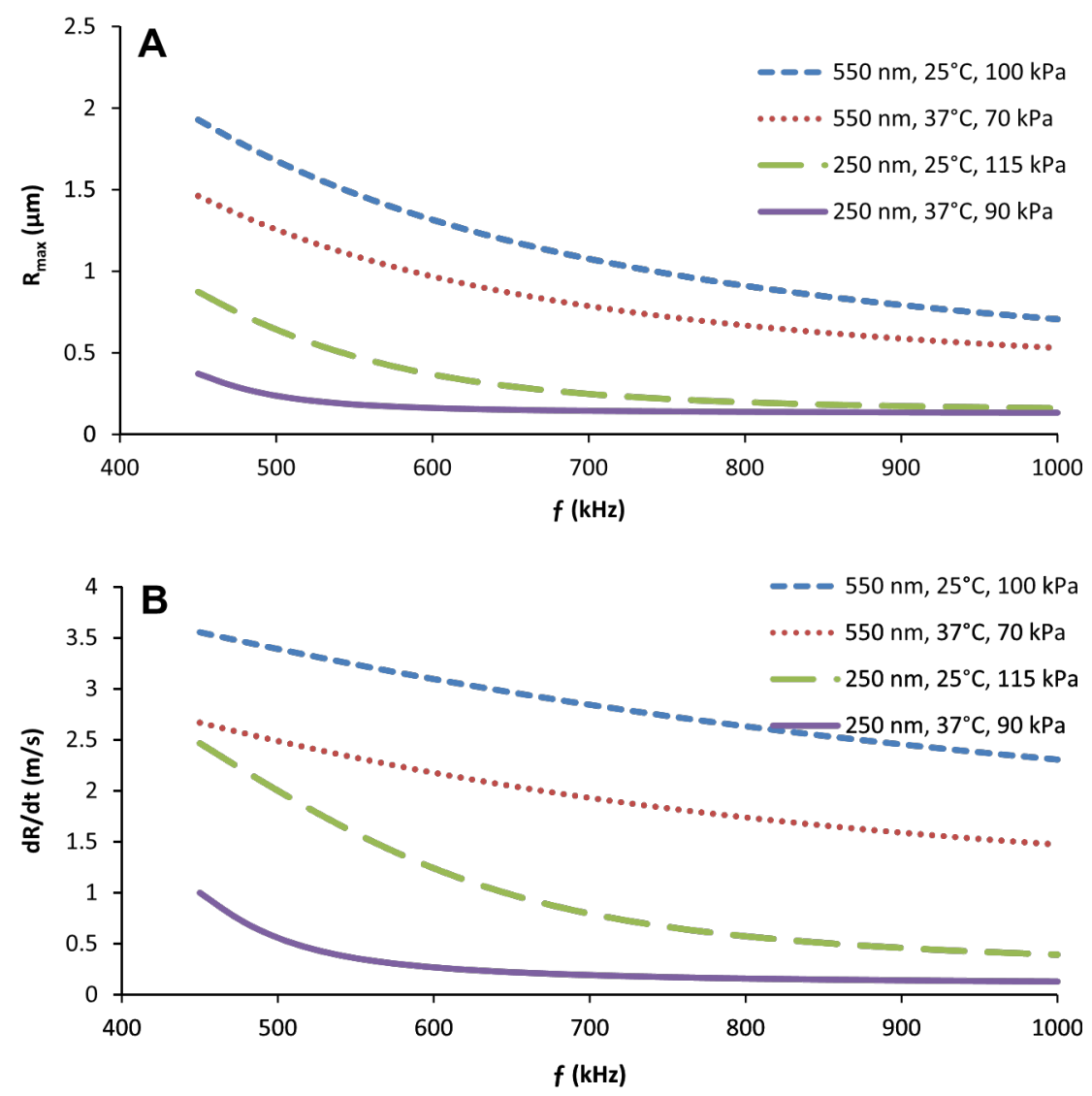

Fig. 10. Effect of US frequency, size and temperature on maximum radius (A) and collapse velocity (B) of the gas bubble. The 250-nm and 550-nm diameter PFC 6 emulsion droplets at $25 \mathrm{C}$ were subject to acoustic amplitudes of $115 \mathrm{kPa}$ and $100 \mathrm{kPa}$, at to $90 \mathrm{kPa}$ and $70 \mathrm{kPa}$ at $37 \mathrm{C}$, respectively. Surface tension is $3.5 \mathrm{mN} / \mathrm{m}$.

cannot form. As mentioned previously, when the surface tension increases there is a longer delay before gas forms, so the bubble does not grow as large during a cycle. The effect of surface tension is shown in Fig. 11 in which a 250-nm PFC 6 emulsion droplet 
is exposed to $115-\mathrm{kPa}$ acoustic amplitude at $25 \mathrm{C}$ and $500 \mathrm{kHz}$. The maximum bubble size decreases almost linearly with increasing surface tension. The same behavior is observed for $\mathrm{PFC}_{5}$. We attribute this to a nearly linear change in the time required for gas phase nucleation since the Laplace pressure is proportional to surface tension (see Eq. (3)). An interfacial tension of $0.004 \mathrm{~N} / \mathrm{m}$ is typical for polar or charged organic compounds adjacent to water [54]. For example, the surface tension of perfluorocarbon in water stabilized by dipalmitoylphosphatidylcholine (DPPC) is estimated to be $0.0035 \mathrm{~N} / \mathrm{m}$ [49].

As Fig. 12 shows, temperature has a very significant effect upon emulsion expansion since the perfluorocarbon vapor pressure is very sensitive to temperature. At lower temperatures, there is not enough vapor pressure to overcome the Laplace pressure. However, once that threshold barrier is overcome, the maximum bubble radius increases very linearly with temperature. The temperature of the expansion threshold is a function of the acoustic amplitude, and for the data set collected, as the acoustic amplitude decreases, the slope of the linear region increases. This is attributed to the observation that the change in vapor pressure with temperature $\left(\mathrm{dP}_{\mathrm{vap}} / \mathrm{dT}\right)$ for perfluorocarbons is larger at $298 \mathrm{~K}$ than at $310 \mathrm{~K}$. Thus at $310 \mathrm{~K}$, the driving force for outward movement of the bubble wall (the difference between the vapor pressure and the local pressure) becomes greater as temperature increases, compared to a slightly smaller driving force change with temperature at $298 \mathrm{~K}$ (see Eq. (32)).

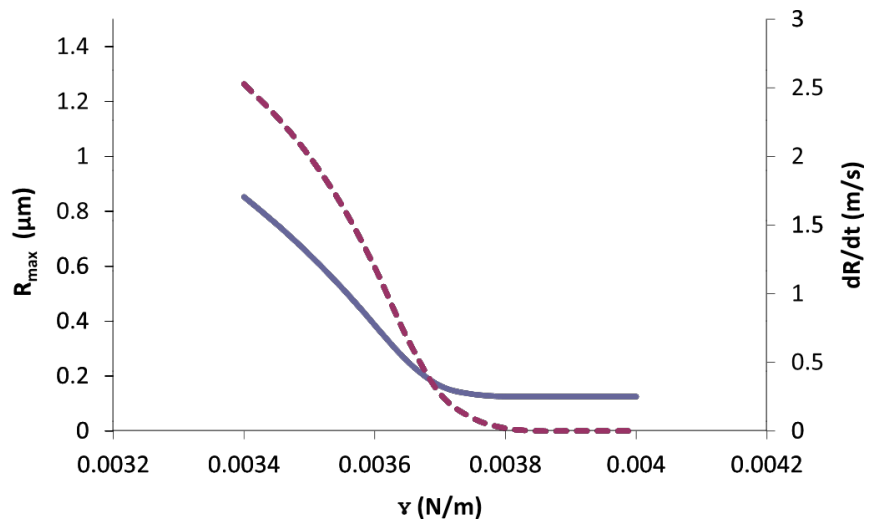

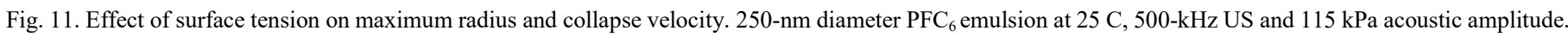

\subsection{Dynamics of emulsion behavior at $20 \mathrm{kHz}$}

When the model was applied to nanoemulsions exposed to $20-\mathrm{kHz}$ ultrasound, the bubble expansion dynamics followed in general the same trends as observed for 500-kHz US, with respect to the influence of initial temperature, initial emulsion droplet size, perfluorocarbon species, and interfacial tension. The general shape of the expansion and collapse is similar to the shape at $500 \mathrm{kHz}$. As an example, Fig. 13 shows the effect of acoustic amplitude on the expansion of $\mathrm{PFC}_{6}$ emulsions at $20 \mathrm{kHz}$ and 25 C. 


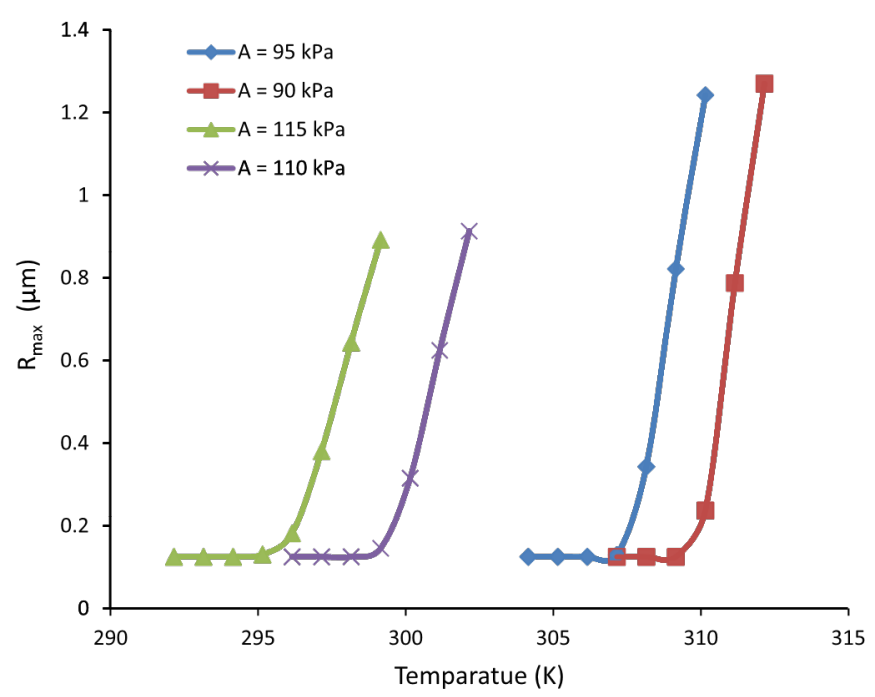

Fig. 12. Effect of initial temperature on $\mathrm{PFC}_{6}$ droplet expansion. 250-nm emulsion droplets were subject to $500 \mathrm{kHz}$ US with $3.5 \mathrm{mN} / \mathrm{m}$ surface tension. The various acoustic amplitudes were $115 \mathrm{kPa}$ (triangles), $110 \mathrm{kPa}(\mathrm{X}), 95 \mathrm{kPa}$ (diamond) and $90 \mathrm{kPa}$ (square).

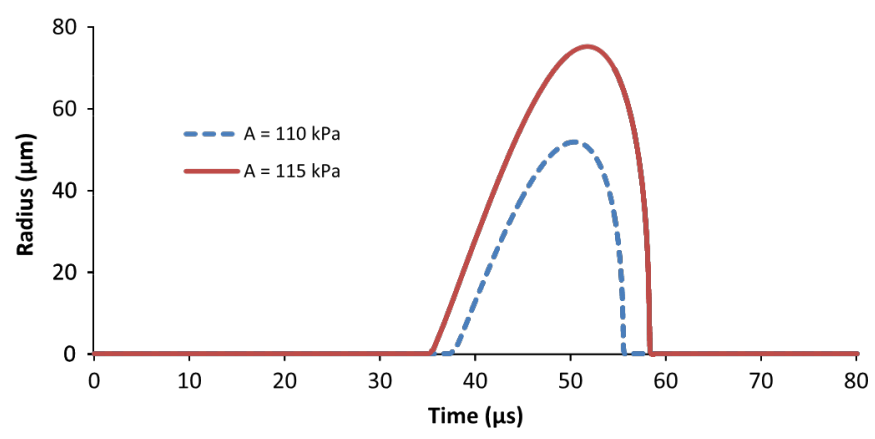

Fig. 13. Effect of acoustic amplitude on $\mathrm{PFC}_{6}$ bubble dynamics at $20 \mathrm{kHz}$. The $250 \mathrm{~nm}$ droplets of $\mathrm{PFC}_{6}$ were subject to $20 \mathrm{kHz}$ US with $3.5 \mathrm{mN} / \mathrm{m}$ surface tension at $25 \mathrm{C}$. The various acoustic amplitudes were $115 \mathrm{kPa}$ (solid line) and $110 \mathrm{kPa}$ (dashed line).

One very noticeable difference is that at $20 \mathrm{kHz}$ the gas phase grew to very large sizes, such as nearly $80 \mathrm{~lm}$ (see Fig. 13). This is attributed to the much longer period of subpressurization in which the bubble can grow. A maximum size on the order of tens of microns indicates that the liquid phase totally evaporates early in the cycle and then recondenses at the end of the collapse. The collapse velocities from such large bubbles, predicted by our simplified code, in some cases exceed the speed of sound in the gas phase, and thus are unrealistic and are not reported herein. Additional terms that account for compressibility in the gas phase need to be incorporated into the model. Such equations are documented [55] and will be incorporated in future versions of the mathematical model. We feel that the current model accurately predicts the expansion dynamics and $\mathrm{R}_{\max }$ values because compressibility effects are not significant during bubble expansion.

Fig. 14 shows the profile of the expansion and collapse of $\mathrm{PFC}_{6}$ emulsions at $25 \mathrm{C}$ over a wide range of frequencies, from 20 $\mathrm{kHz}$ to $500 \mathrm{kHz}$. Each expansion and collapse appears shifted because at each different frequency, there is a different time delay before the gas phase nucleates. The expansion at $500 \mathrm{kHz}$ is extremely small, and barely perceptible on the same plot with the expansion at $20 \mathrm{kHz}$. Fig. 14 illustrates the enormous bubble size that is attained at the lower frequencies. Obviously much more mechanical stress due to cavitation will occur to cells or drug carriers in the vicinity of phase changing emulsions at lower frequencies than at higher frequencies. 


\section{Discussion}

We have constructed a mathematical model that captures the essentials of the heat, mass and momentum transport phenomena that occurs during acoustic droplet vaporization. While the general qualitative effects of droplet composition, size, temperature and surface tension are predictable, this model verified the predictions and provided intricate details of bubble formation and collapse.

From the results shown by the mathematical model, it can be concluded that in general a larger emulsion droplet grows into a larger sized gas bubble. This is because the Laplace pressure is smaller in the larger emulsion droplet, and the gas evolution is initiated earlier in the acoustic cycle. This provides a longer time of subpressurization and a larger subpressurization driving force,

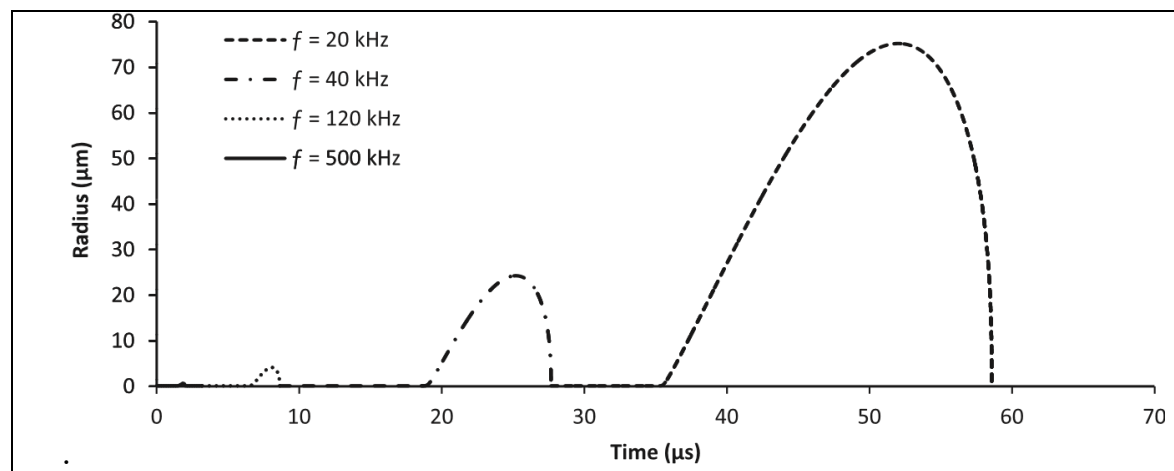

Fig. 14. Effect of frequency on $\mathrm{PFC}_{6}$ emulsion droplet expansion. All emulsions were $\mathrm{PFC}_{6}$ droplets of 250 -nm diameter at $25 \mathrm{C}$ and a surface tension of $3.5 \mathrm{mN}$ subject to $110 \mathrm{kPa}$ insonation.

both of which contribute to expansion. Similarly, decreasing the frequency also provides more time for expansion and creates larger bubbles. A larger emulsion producing a larger bubble has a larger collapse velocity compared to the collapse velocity of a bubble from a smaller nanodroplet. Furthermore, our numerical model showed (see Fig. 8) that the radius of the bubble increases fairly linearly with acoustic amplitude, and the bubble collapse velocity showed an increasing linear trend with acoustic amplitude. The bubble size also increases fairly linearly with temperature, once the nucleation threshold is attained Upon increasing the surface tension, there was a fairly linear decrease in maximum bubble radius once the threshold of gas formation was achieved. The maximum radius decreases linearly with increasing surface tension due to a shorter subpressurization time and an increase in the Laplace pressure that "squeezes" the bubble.

The bubble collapse velocity decreases with increasing surface tension because the bubble grows to a smaller size.

Both perfluorocarbons show that the maximum bubble radius increases with temperature and amplitude. Our numerical model shows that the collapse velocity is fairly sensitive to US parameters of frequency, amplitude, to temperature, and to the surface tension and initial droplet size. This observation is noteworthy since the collapse velocity may have the largest influence on cells and drug delivery vehicles.

The emulsion expansion was very sensitive to the frequency of insonation (see Fig. 14). The influence of ultrasonic frequency on the biological effects of cavitating gas bubbles is well documented in that biological effects are much more prominent at lower frequencies than higher frequencies [56-59]. Many of these effects

are associated with inertial cavitation, whose onset is correlated by the Mechanical Index parameter: MI $=(\mathrm{A} / \mathrm{MPa}) /(\mathrm{f} / \mathrm{MHz})$. $[56,57,60]$ At this time we do not have enough data to determine whether bubble size or collapse velocity also correlates with the inverse square root of frequency, but we can propose that mechanical effects will increase as frequency decreases.

Two mathematical models were developed to manage the heat transfer to and from the droplet as evaporation and condensation occurred: a boundary layer thermal model and a constant temperature model. These were bracketing asymptotic models. On comparison of these two models (see Fig. 4), the surface temperatures differed at most by a few degrees, and these small changes 
were not sufficient to produce any significant changes to the mechanical expansion and contraction of the gas phase, at least within the ranges of the parameters explored in this study. Thus either model may be sufficiently accurate to provide a general picture of bubble behavior.

As for practical applications of this model of acoustic droplet vaporization, the results indicate that if mechanical movement and stress is the desired outcome, low frequency ultrasound will produce enormous gas expansion even after the liquid PFC has completely vaporized. The shear stresses generated by such expansions can perturb nearby cells and perhaps drug carriers, even at fairly low acoustic amplitudes. However, if gas formation without great expansion is desired, such as in producing contrast for ultrasonic imaging, high frequencies would be better. At high frequencies, gas may form, but there is not much time for expansion.

The model also showed that it may be experimentally very difficult to "dial in" the exact acoustic amplitude to obtain partial vaporization. While temperature and interfacial tension have a strong influence on emulsion expansion, they cannot be easily manipulated in a clinical setting, leaving only emulsion composition, acoustic pressure amplitude, and emulsion size and variables that can be tweaked to achieve a given expansion. Given that most emulsions have a size distribution, and the window between no gas formation and full vaporization is narrow, and the expansion is fairly sensitive to the initial size of the emulsion droplet, there may be some emulsion droplets in a distribution that do not form any gas, and others that become fully vaporized and form large bubbles. We surmise that precise control of expansion will depend upon precise control of emulsion size distribution, as well as careful acoustic power delivery.

\section{Conclusions}

A mathematical model of the vaporization and re-condensation of PFC liquid droplets was used to predict the bubble behavior of $\mathrm{PFC}_{5}$ and $\mathrm{PFC}_{6}$ nanoemulsions. The calculations were executed using a Runga-Kutta algorithm to predict bubble dynamics as a function of ultrasonic frequency, pressure amplitude, bubble size and interfacial tension. Some of the simplifying assumptions in the model are that there are no pressure gradients in the gas phase, and that temperature gradients are linear, that the composition of the gas phase is not temperature dependent and that the droplet and bubble were concentric. A major assumption is that the gas phase forms once the local pressure drops below the vapor pressure of the PFC. In actuality, nucleation may take some time or some subpressurization, and may not occur in each acoustic cycle, although corresponding physical experiments indicate that nucleation of a gas phase occurs, and can occur quickly in experiments with large subpressurizations.

With respect to the size of the nanoemulsion, the results indicate that larger droplets form larger gas bubbles, and commence gas formation earlier in the acoustic cycle. This is attributed to the role of surface tension. With respect to PFC composition, $\mathrm{PFC}_{5}$ with the higher vapor pressure forms larger bubbles. Likewise, increasing temperature increases vapor pressure and produces larger bubbles. Larger bubbles lead to larger collapse velocities.

The frequency has a profound effect, as the bubble dynamics appeared to be dominated by the momentum of the water. At $500 \mathrm{kHz}$, the bubble oscillation was fast and there was not much time for the water to gain much momentum, which resulted in small expansion at the acoustic amplitudes used in this model. However, at $20 \mathrm{kHz}$ and the same acoustic amplitude, the bubble expansion was orders of magnitude larger, with complete evaporation of the PFC liquid droplet. This is attributed to the longer time of expansion, which continued to increase the momentum of the water, so that the bubble continued to expand even after the pressure driving force was no longer favorable.

We feel that this research has only opened the door to modeling the dynamics of phase transformation of liquid droplets to gas bubbles. More research and more detailed models remain to be developed that include more sophisticated models than the Rayleigh-Plesset equation, especially as bubble wall velocities approach the speed of sound and the interior gas is compressed. 
Of critical import is a better understanding of nucleation phenomena, which has not been included in this model. Armed with these modeling tools, we may be better able to design phase-changing nanoemulsions and coordination with ultrasonic exposure to produce a gas bubble that will create a designed biological or imaging effect.

\section{References}

[1] G.A. Husseini, W.G. Pitt, Micelles and nanoparticles in ultrasonic drug and gene delivery, Advanced Drug Delivery Reviews 60 (2008) $1137-1152$.

[2] K. Hynynen, Ultrasound for drug and gene delivery to the brain, Advanced Drug Delivery Reviews 60 (2008) $1209-1217$.

[3] C.R. Mayer, R. Bekeredjian, Ultrasonic gene and drug delivery to the cardiovascular system, Advanced Drug Delivery Reviews 60 (2008) $1177-1192$.

[4] V. Frenkel, Ultrasound mediated delivery of drugs and genes to solid tumors, Advanced Drug Delivery Reviews 60 (2008) $1193-1208$.

[5] J.R. Wu, W.L. Nyborg, Ultrasound, cavitation bubbles and their interaction with cells, Advanced Drug Delivery Reviews 60 (2008) $1103-1116$.

[6] N.Y. Rapoport, A.M. Kennedy, J.E. Shea, C.L. Scaife, K.H. Nam, Controlled and targeted tumor chemotherapy by ultrasound-activated nanoemulsions/ microbubbles, Journal of Controlled Release 138 (2009) 268-276.

[7] A. Schroeder, J. Kost, Y. Barenholz, Ultrasound, liposomes, and drug delivery: principles for using ultrasound to control the release of drugs from liposomes, Chemistry and Physics of Lipids 162 (2009) 1-16.

[8] M.L. Fabiilli, K.J. Haworth, I.E. Sebastian, O.D. Kripfgans, P.L. Carson, J.B. Fowlkes, Delivery of chlorambucil using an acoustically-triggered perfluoropentane emulsion, Ultrasound in Medicine and Biology 36 (2010) 1364-1375.

[9] C.S. Yoon, J.H. Park, Ultrasound-mediated gene delivery, Expert Opinion on Drug Delivery 7 (2010) 321-330.

[10] P.S. Sheeran, S. Luois, P.A. Dayton, T.O. Matsunaga, Formulation and acoustic studies of a new phase-shift agent for diagnostic and therapeutic ultrasound, Langmuir: The ACS Journal of Surfaces and Colloids 27 (2011) 10412-10420.

[11] M. Seidl, P. Steinbach, K. Worle, F. Hofstadter, Induction of stress fibers and intercellular gaps in human vascular endothelium by shock-waves, Ultrasonics 32 (1994) 397-400.

[12] A.H. Mesiwala, L. Farrell, H.J. Wenzel, D.L. Silbergeld, L.A. Crum, H.R. Winn, P.D. Mourad, High-intensity focused ultrasound selectively disrupts the bloodbrain barrier in vivo, Ultrasound in Medicine and Biology 28 (2002) 389-400.

[13] V. Frenkel, A. Etherington, M. Greene, J. Quijano, J.W. Xie, F. Hunter, S. Dromi, K.C.P. Li, Delivery of liposomal doxorubicin (Doxil) in a breast cancer tumor model: investigation of potential enhancement by pulsed-high intensity focused ultrasound exposure, Academic Radiology 13 (2006) $469-479$.

[14] S.M. Stieger, C.F. Caskey, R.H. Adamson, S.P. Qin, F.R.E. Curry, E.R. Wisner, K.W. Ferrara, Enhancement of vascular permeability with low-frequency contrastenhanced ultrasound in the chorioallantoic membrane model, Radiology 243 (2007) 112-121.

[15] Z. Gao, A.M. Kennedy, D.A. Christensen, N.Y. Rapoport, Drug-loaded nano/ microbubbles for combining ultrasonography and targeted chemotherapy, Ultrasonics 48 (2008) 260-270.

[16] M.R. Bohmer, C.H.T. Chlon, B.I. Raju, C.T. Chin, T. Shevchenko, A.L. Klibanov, Focused ultrasound and microbubbles for enhanced extravasation, Journal of Controlled Release 148 (2010) 18-24.

[17] C.-Y. Lin, T.-M. Liu, C.-Y. Chen, Y.-L. Huang, W.-K. Huang, C.-K. Sun, F.-H. Chang, W.-L. Lin, Quantitative and qualitative investigation into the impact of focused ultrasound with microbubbles on the triggered release of nanoparticles from vasculature in mouse tumors, Journal of Controlled Release 146 (2010) 291- 298 .

[18] R.K. Schlicher, H. Radhakrishna, T.P. Tolentino, R.P. Apkarian, V. Zarnitsyn, M.R. Prausnitz, Mechanism of intracellular delivery by acoustic cavitation, Ultrasound in Medicine and Biology 32 (2006) 915-924.

[19] P. Prentice, A. Cuschierp, K. Dholakia, M. Prausnitz, P. Campbell, Membrane disruption by optically controlled microbubble cavitation, Nature Physics 1 (2005) 107-110.

[20] D.M. Hallow, R.A. Seeger, P.P. Kamaev, G.R. Prado, M.C. LaPlaca, M.R. Prausnitz, Shear-induced intracellular loading of cells with molecules by controlled microfluidics, Biotechnology and Bioengineering 99 (2008) 846-854.

[21] Z. Fan, R.E. Kumon, J. Park, C.X. Deng, Intracellular delivery and calcium transients generated in sonoporation facilitated by microbubbles, Journal of Controlled Release: Official Journal of the Controlled Release Society 142 (2010) 31-39.

[22] A. van Wamel, K. Kooiman, M. Harteveld, M. Emmer, F.J. ten Cate, M. Versluis, N. de Jong, Vibrating microbubbles poking individual cells: drug transfer into cells via sonoporation, Journal of Controlled Release 112 (2006) 149-155.

[23] W.G. Pitt, G.A. Husseini, Ultrasound in drug and gene delivery, Advanced Drug Delivery Reviews 60 (2008) 1095-1096.

[24] S.K. Hobbs, W.L. Monsky, F. Yuan, W.G. Roberts, L. Griffith, V.P. Torchilin, R.K. Jain, Regulation of transport pathways in tumor vessels: role of tumor type and microenvironment, Proceedings of the National Academy of Sciences of the United States of America 95 (1998) 4607-4612. 
[25] L.W. Seymour, Passive tumor targeting of soluble macromolecules and drug conjugates, Critical Reviews in Therapeutic Drug Carrier Systems 9 (1992) $135-187$.

[26] M. Kissel, P. Peschke, V. Subr, K. Ulbrich, J. Schuhmacher, J. Debus, E. Friedrich, Synthetic macromolecular drug carriers: biodistribution of poly[(N2hydroxypropyl)methacrylamide] copolymers and their accumulation in solid rat tumors, PDA Journal of Pharmaceutical Science and Technology/PDA 55 (2001) 191-201.

[27] D.L. Miller, O.D. Kripfgans, J.B. Fowlkes, P.L. Carson, Cavitation nucleation agents for nonthermal ultrasound therapy, Journal of the Acoustical Society of America 107 (2000) 3480-3486.

[28] O.D. Kripfgans, J.B. Fowlkes, D.L. Miller, O.P. Eldevik, P.L. Carson, Acoustic droplet vaporization for therapeutic and diagnostic applications, Ultrasound in Medicine and Biology 26 (2000) 1177-1189.

[29] N. Rapoport, Z.G. Gao, A. Kennedy, Multifunctional nanoparticles for combining ultrasonic tumor imaging and targeted chemotherapy, Journal of the National Cancer Institute 99 (2007) 1095-1106.

[30] N. Rapoport, K.H. Nam, R. Gupta, Z.G. Gao, P. Mohan, A. Payne, N. Todd, X. Liu, T. Kim, J. Shea, C. Scaife, D.L. Parker, E.K. Jeong, A.M. Kennedy, Ultrasoundmediated tumor imaging and nanotherapy using drug loaded, block copolymer stabilized perfluorocarbon nanoemulsions, Journal of Controlled Release 153 (2011) 4-15.

[31] P.S. Sheeran, S.H. Luois, L.B. Mullin, T.O. Matsunaga, P.A. Dayton, Design of ultrasonically-activatable nanoparticles using low boiling point perfluorocarbons, Biomaterials 33 (2012) 3262-3269.

[32] J.R. Lattin, D.M. Belnap, W.G. Pitt, Formation of eLiposomes as a drug delivery vehicle, Colloids and Surfaces B 89 (2012) $93-100$.

[33] M. Javadi, W.G. Pitt, D.M. Belnap, N.H. Tsosie, J.M. Hartley, Encapsulating emulsions inside liposomes for drug delivery, Langmuir 28 (2012) 1472014729.

[34] M. Javadi, W.G. Pitt, C.M. Tracy, J.R. Barrow, B.M. Willardson, J.M. Hartley, N.H. Tsosie, Ultrasonic gene and drug delivery using eLiposomes, Journal of Controlled Release 167 (2013) 92-100.

[35] M.L. Fabiilli, K.J. Haworth, N.H. Fakhri, O.D. Kripfgans, P.L. Carson, J.B. Fowlkes, The role of inertial cavitation in acoustic droplet vaporization, IEEE Transactions on Ultrasonics, Ferroelectrics, and Frequency Control 56 (2009) 1006-1017.

[36] M. Zhang, M. Fabiilli, P. Carson, F. Padilla, S. Swanson, O. Kripfgans, B. Fowlkes, Acoustic droplet vaporization for the enhancement of ultrasound thermal therapy, Proceedings of the IEEE Ultrasonics Symposium 2010 (2010) 221- 224.

[37] C.-Y. Lin, W.G. Pitt, Acoustic droplet vaporization in biology and medicine, BioMed Research International (2013). submitted for publication.

[38] Z.Z. Wong, O.D. Kripfgans, A. Qamar, J.B. Fowlkes, J.L. Bull, Bubble evolution in acoustic droplet vaporization at physiological temperature via ultrahigh speed imaging, Soft Matter 7 (2011) 4009-4016.

[39] K.J. Haworth, J.B. Fowlkes, P.L. Carson, O.D. Kripfgans, Towards aberration correction of transcranial ultrasound using acoustic droplet vaporization, Ultrasound in Medicine and Biology 34 (2008) 435-445.

[40] S.T. Kang, C.K. Yeh, Intracellular acoustic droplet vaporization in a single peritoneal macrophage for drug delivery applications, Langmuir: The ACS Journal of Surfaces and Colloids 27 (2011) 13183-13188.

[41] P.S. Bapat, A.B. Pandit, Thermodynamic and kinetic considerations of nucleation and stabilization of acoustic cavitation bubbles in water, Ultrasonics Sonochemistry 15 (2008) 65-77.

[42] R.L. Rowley, W.V. Wilding, J.L. Oscarson, Y. Yang, N.F. Giles, DIPPR Data Compilation of Pure Chemical Properties, in: Design Institute for Physical Properties, AIChE, New York, NY, 2008.

[43] O. Couture, P.D. Bevan, E. Cherin, K. Cheung, P.N. Burns, F.S. Foster, Investigating perfluorohexane particles with high-frequency ultrasound, Ultrasound in Medicine and Biology 32 (2006) 73-82.

[44] T. Giesecke, K. Hynynen, Ultrasound-mediated cavitation thresholds of liquid perfluorocarbon droplets in vitro, Ultrasound in Medicine and Biology 29 (2003) 1359-1365.

[45] S. Podell, B. Golec, R. Lohrmann, Measuring the effects of ultrasound on contrast agents, in: Ieee T Ultrason Ferr, IEEE, 1999 , pp. 1749-1754.

[46] P.S. Sheeran, V.P. Wong, S. Luois, R.J. McFarland, W.D. Ross, S. Feingold, T.O. Matsunaga, P.A. Dayton, Decafluorobutane as a phase-change contrast agent for low-energy extravascular ultrasonic imaging, Ultrasound in Medicine and Biology 37 (2011) 1518-1530.

[47] O.D. Kripfgans, M.L. Fabiilli, P.L. Carson, J.B. Fowlkes, On the acoustic vaporization of micrometer-sized droplets, Journal of the Acoustical Society of America 116 (2004) 272-281.

[48] R. Singh, G.A. Husseini, W.G. Pitt, Phase transitions of nanoemulsions using ultrasound: experimental measurements, Ultrasonics Sonochemistry 19 (2012) 1120-1125.

[49] A. Kabalnov, J. Weers, R. Arlauskas, T. Tarara, Phospholipids as emulsion stabilizers. 1. Interfacial tensions, Langmuir 11 (1995) $2966-2974$.

[50] R.B. Bird, W.E. Stewart, E.N. Lightfoot, Transport Phenomena, Second ed., Wiley, New York, 2007. 
D.A. Christensen, Ultrasonic Bioinstrumentation, Wiley, New York, 1988.

[52] M.A. Diaz de la Rosa, W.G. Pitt, G.A. Husseini, Mathematical modeling of microbubble cavitation at $70 \mathrm{kHz}$ and the importance of the subharmonic in drug delivery from micelles, Ultrasonics 53 (2013) 97-110.

[53] M.A. Diaz de la Rosa, W.G. Pitt, G.A. Husseini, Comparing microbubble cavitation at $500 \mathrm{kHz}$ and $70 \mathrm{kHz}$ related to micellar drug delivery using ultrasound, Ultrasonics (2012). accepted.

[54] D. Needham, R.S. Nunn, Elastic-deformation and failure of lipid bilayermembranes containing cholesterol, Biophysical Journal 58 (1990) 997-1009.

[55] C.E. Brennen, Cavitation and Bubble Dynamics, Oxford University Press, New York, 1995.

[56] S. Barnett, Thresholds for nonthermal biofeffects: theoretical and experimental basis for a threshold index, Ultrasound in Medicine and Biology 24 (1998) S41-S49.

[57] W.L. Nyborg, Biological effects of ultrasound: development of safety guidelines. Part II: general review, Ultrasound in Medicine and Biology 27 (2001) $301-333$.

[58] G. Korosoglou, S.E. Hardt, R. Bekeredjian, J. Jenne, M. Konstantin, M. Hagenmueller, H.A. Katus, H. Kuecherer, Ultrasound exposure can increase the membrane permeability of human neutrophil granulocytes containing microbubbles without causing complete cell destruction, Ultrasound in Medicine and Biology 32 (2006) 297-303.

[59] D.L. Miller, R.M. Thomas, Thresholds for hemorrhages in mouse skin and intestine induced by lithotripter shock waves, Ultrasound in Medicine and Biology 21 (1995) 249-257.

[60] R.E. Apfel, C.K. Holland, Gauging the liklihood of cavitation from short-pulse, low-duty cycle diagnostic ultrasound, Ultrasound in Medicine and Biology 17 (1991) 179-185. 